\title{
AN EXPOSITION OF THE STRUCTURE OF SOLVMANIFOLDS. PART I: ALGEBRAIC THEORY ${ }^{1}$
}

\author{
BY LOUIS AUSLANDER ${ }^{2}$
}

CONTENTS

\section{PrefaCE}

Chapter I. Abelian theOry.

ChaPTER II. NilPotent LiE GROUPS AND NILMANIFOLDS.

1. What is a nilpotent Lie group?

2. Global nilpotent theory.

3. Rational nilpotent groups.

4. Compact nilmanifolds.

ChaPTER III. SOlVABLE LIE GROUPS AND SOLVMANIFOLDS.

1. Examples.

2. The structure of solvable Lie groups-the splitting theorems.

3. Splitting theorems and a global theorem concerning solvmanifolds.

4. Abstract splitting theorems and uniqueness of compact solvmanifolds.

5. Existence of compact solvmanifolds.

6. Presentations of compact solvmanifolds.

7. Compact solvmanifolds with discrete isotropy groups.

CHAPTER IV. DISCRETE SEMISIMPLE SPLITTINGS AND APPLICATIONS.

1. Introduction.

2. Discrete semisimple splittings.

3. The P. Hall conjecture and the Mostow structure theorem for presentations of compact solvmanifolds.

4. Solvable Lie groups of exponential type.

5. Type $R$ solvmanifolds and their generalizations.

\section{REFERENCES}

AMS (MOS) subject classifications (1970). Primary 22 E25.

${ }^{1}$ The material in this article represents an enlargement of part of the material the author presented in his address to the Society at its Annual Meeting in San Francisco in 1968; received by the editors June 11, 1972.

${ }^{2}$ John Simon Guggenheim Fellow and partially supported by grant from National Science Foundation. 


\section{PREFACE}

The modern theory of solvmanifolds began in 1949 with the beautiful and fundamental paper of A. Malcev [M, A;1]. In the past 20 years or so the role played by algebraic group theory in establishing the basic structure theorems of solvmanifolds has become more and more apparent. In this exposition we have used algebraic groups as our basic tool in presenting a unified account of solvable Lie groups and solvmanifolds. Although there are few new theorems in this paper, many of the proofs given here will appear in print for the first time.

The general reference for algebraic groups will be $\mathrm{A}$. Borel [B, A] and for Lie algebras will be $\mathrm{N}$. Jacobson [J, N]. In addition we will use the following general facts and notation throughout this paper:

Let $G$ be a connected, simply connected Lie group. We will denote its Lie algebra by $L(G)$. The group of automorphisms of $G$ and $L(G)$ will be denoted by $A(G)$ and $A(L(G))$, respectively. Then $A(G)$ and $A(L(G))$ are canonically isomorphic. Further $A(L(G)) \subset G L(L(G))$ is an algebraic group. Thus if we talk of an automorphism $\theta$ of $G$ being semisimple or unipotent we will mean that the image of $\theta$ in $A(L(G))$ is semisimple or unipotent. Further if $\theta \in A(G)$, by the semisimple or unipotent parts of $\theta$, we will mean the semisimple or unipotent part of $\theta$ when viewed in $A(L(G))$ and since $A(L(G))$ is algebraic we know that the semisimple and unipotent parts of $\theta$ are also automorphisms of $G$. If $\Phi$ is a subgroup of an algebraic group $G \subset G L(n, R)$ by the algebraic hull of $\Phi$, denoted by $A_{h}(\Phi)$, we will mean the intersection of all the algebraic subgroups of $G L(n, R)$ containing $\Phi$. Note that $A_{h}(\Phi) \subset G$ and is algebraic.

\section{CHAPTER I : ABELIAN THEORY}

Although the abelian theory is by now classical, let us quickly run through it as a way of setting the stage for the generalizations to come.

Let $\boldsymbol{R}^{n}$ be a connected, simply connected, abelian Lie group and let $H$ be a closed subgroup of $\boldsymbol{R}^{n}$. We will also view $\boldsymbol{R}^{n}$ as an algebraic group of the form

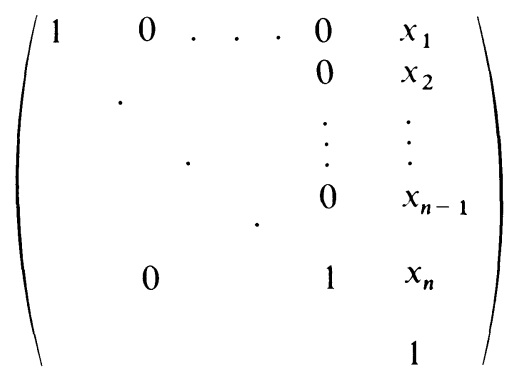

in $G L(n+1, R)$. Note that viewing $\boldsymbol{R}^{n}$ as an algebraic group in this manner 
has the important consequence that the algebraic subgroups of $\boldsymbol{R}^{n}$ are exactly the linear subspaces of $\boldsymbol{R}^{n}$. Thus if $H \subset \boldsymbol{R}^{n}$ we may talk either of the linear span of $H$ or the algebraic hull of $H, A_{h}(H)$. We will now briefly outline how we will think about the structure of $\boldsymbol{R}^{n} / H$.

Global theory. Let $H_{0}$ denote the identity component of $H$ and let $A_{h}(H)$ be the linear span of $H$ or the algebraic hull of $H$. Then $H_{0}$ is a connected normal subgroup of $A_{h}(H)$ and $A_{h}(H) / H$ is a compact abelian group or torus whose fundamental group is $H / H_{0}$. Further since $\boldsymbol{R}^{n}=V \oplus A_{h}(H)$, where $V$ is a vector subspace $\boldsymbol{R}^{n} / H \approx V \times A_{h}(H) / H \approx$ $V \times T$, where $T$ is the torus $A_{h}(H) / H$. Thus $R^{n} / H$ is compact if and only if $\boldsymbol{R}^{n}=A_{h}(H)$.

Rational theory. Let $e_{1}, \ldots, e_{n}$ be a basis for $\boldsymbol{R}^{n}$ and let $\boldsymbol{Q}^{n} \subset \boldsymbol{R}^{n}$ be the subgroup of all rational linear combinations of this basis. We will call $\boldsymbol{Q}^{n}$ a rational form of $\boldsymbol{R}^{n}$. Let $G$ be a subgroup of $\boldsymbol{Q}^{n}$. It is well known that $G$ is a discrete subgroup of $\boldsymbol{R}^{n}$ if and only if $G$ is finitely generated. Now let $\pi$ be a finitely generated subgroup of $Q^{n}$ and let $A_{h}(\pi, Q)$ be the rational subspace of $Q^{n}$ spanned by $\pi$, or, equivalently, all the rational points of $A_{h}(\pi) \subset \boldsymbol{R}^{n}$. Further, let $W$ be any rational subspace of $\boldsymbol{Q}^{n}$. Then

$$
A_{h}(\pi \cap W, Q)=A_{h}(\pi, Q) \cap W .
$$

Compact theory. Let $\pi \subset \boldsymbol{R}^{m}$ be a discrete subgroup of $\boldsymbol{R}^{m}$ (or, equivalently, a finitely generated subgroup of a rational form of $\boldsymbol{R}^{m}$ ) such that $A_{h}(\pi)=\boldsymbol{R}^{m}$. Let $\pi_{1} \subset \boldsymbol{R}^{n}$ also be a discrete subgroup of $\boldsymbol{R}^{n}$ such that $A_{h}\left(\pi_{1}\right)=\boldsymbol{R}^{n}$. If $\psi: \pi \rightarrow \pi_{1}$ is an isomorphism then $\psi$ can be extended uniquely to an isomorphism $\psi^{*}: \boldsymbol{R}^{m} \rightarrow \boldsymbol{R}^{n}$. Hence $m=n$ and $\psi^{*}$ induces a diffeomorphism of $\boldsymbol{R}^{n} / \pi$ and $\boldsymbol{R}^{m} / \pi_{1}$.

\section{ChAPTER II : NilPOTENT LIE GROUPS AND NILMANIFOLDS}

1. What is a nilpotent Lie group? Classically, a connected, simply connected Lie group $N$ is said to be nilpotent if the Lie algebra of $N, L(N)$, is a nilpotent Lie algebra. This is then shown to be equivalent to requiring that $N$ is nilpotent as an abstract group. (Recall that an abstract group is said to be nilpotent if its lower central series is finite. This amounts to requiring that if $G_{k}$ is defined inductively by $G_{1}=G$ and $\left[G_{k-1}, G\right]=G_{k}$, where $[H, K]$ is the group generated by all elements of the form $k h k^{-1} h^{-1}$, $k \in K, h \in H$, then $G_{k}=e$ for some finite $k$, where $e$ is the identity element of $G$.)

It is a consequence of the Birkhoff imbedding theorem, $[B, G]^{3}$, that we may find an isomorphism of any connected, simply connected nilpotent Lie group $N$ into the upper triangular unipotent matrix group, $U(m)$, where

\footnotetext{
${ }^{3}$ See references at end of paper.
} 


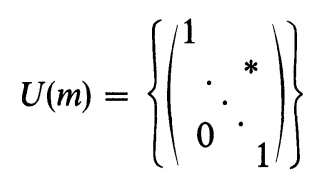

is a subgroup of $G L(m, R)$, for some $m$. But any connected subgroup of $U(m)$ is easily seen to be an algebraic group. What is also important is that the algebraic subgroups of $N$ for any imbedding in $U(m)$, any $m$, consist exactly of the connected subgroups of $N$. (Notice the similarity with $\boldsymbol{R}^{n}$ from Chapter I.) Thus if $H$ is any subgroup of $N$ we may form the algebraic hull of $H, A_{h}(H)$, in $N$, which is defined as the intersection of all the algebraic subgroups of $N$ that contain $N$ or equivalently the smallest connected subgroup of $N$ containing $H$.

Our ability to represent $N$ faithfully in $U(m)$ also enables us to do the following: We can find a coordinate system $\boldsymbol{X}=\left(x_{1}, \ldots, x_{n}\right)$ in all of $N$ such that multiplication in $N$ is given by

$$
\boldsymbol{X} \cdot \boldsymbol{Y}=\left(x_{1}, \ldots, x_{n}\right) \cdot\left(y_{1}, \ldots, y_{n}\right)=\left(p_{1}(\boldsymbol{X}, \boldsymbol{Y}), \ldots, p_{n}(\boldsymbol{X}, \boldsymbol{Y})\right)
$$

where the $p_{i}, i=1, \ldots, n$, are polynomials in $2 n$ variables. The converse is also true and a proof can be found in $[\mathrm{T}, \mathrm{R} ; \mathbf{5}]$. The converse may be formally stated as follows: Let $N$ be a group with coordinates $\boldsymbol{X}=\left(x_{1}, \ldots, x_{n}\right)$ whose law of composition satisfies equation (1), then $N$ is a nilpotent Lie group.

Henceforth, we will adopt either the point of view that $N$ is an algebraic group or that the group multiplication is polynomial in talking about or working with nilpotent Lie groups. Hence, unless explicitly stated to the contrary nonabelian nilpotent Lie groups will always be connected and simply connected. We will say that a field $\mathscr{F}$ is a field of definition for the nilpotent Lie group $N$ if there exists a polynomial law of composition in $N$ all of whose coefficients are in $\mathscr{F}$ or if $N$ can be represented as an algebraic group with $\mathscr{F}$ as field of definition. We may now talk of the points of $N$ in any field $\mathscr{K}$ containing $\mathscr{F}$; i.e., a point of $N$ in $\mathscr{K}$ is any $n$-tuple $\left(k_{1}, \ldots, k_{n}\right), k_{i} \in \mathscr{K}$. For the rest of this paper we will call a nilpotent Lie group defined over the rational field $\boldsymbol{Q}$ a rational nilpotent Lie group. These will play a particularly important role in our theory.

2. Global nilpotent theory. Let $H$ be a closed subgroup of the nilpotent Lie group $N$ and let $H_{0}$ denote the identity component of $H$. If $A_{h}(H)$ again denotes the algebraic hull of $H$ in $N$ and if $A_{h}(H) \neq N$, it is easily seen that $N / A_{h}(H)$ is topologically a vector space $V$ and that

$$
N / H \approx V \times A_{h}(H) / H
$$

where $\approx$ denotes homeomorphism. 
Let us now examine the case where $H \subset N$ and $A_{h}(H)=N$. We begin by observing that, in this case, $H_{0}$ is a normal subgroup of $N$, because $H_{0}$ is normal in $H$ and $N$ is the algebraic hull of $H$. Thus we may form the nilpotent Lie group $M=N / H_{0}$ and consider $H / H_{0}$ as a discrete subgroup $\pi$ of $M$. It is easily seen that $A_{h}(\pi)=M$. Now, by standard results in the theory of algebraic groups,

$$
A_{h}([\pi, \pi])=\left[A_{h}(\pi), A_{h}(\pi)\right]=[M, M] .
$$

A simple inductive argument now permits us to conclude that $M / \pi \approx N / H$ is compact or we have that if $H$ is a closed subgroup of the nilpotent Lie group $N$ then $N / H$ is compact if and only if $A_{h}(H)=N$. Let us explicitly see that if $A_{h}(H)=N$, then $N / H$ is compact. This amounts to showing that $M / \pi$ is compact and by the above relation and induction we may assume that $[M, M] /[\pi, \pi]$ is compact. From this it follows that the image of $\pi$ in the abelian group $M /[M, M]$ is discrete and, by an easy argument on algebraic or linear hulls, it follows that the image of $\pi$ in $M /[M, M]$ is cocompact or spans the vector space. Hence $M / \pi$ is a fiber bundle with compact fiber $[M, M] /[M, M] \cap \pi$ and compact base $(M / \pi[M, M])$ and so is compact.

Let us now adopt the language of calling the homogeneous space of a nilpotent Lie group a nilmanifold. Then we may summarize our results of this section by the statement that a nilmanifold is the Cartesian product of a vector space and a compact nilmanifold.

3. Rational nilpotent Lie groups. Let $N$ be a nilpotent Lie group and let $\left(x_{1}, \ldots, x_{n}\right)=X$ be a coordinate system in $N$ such that multiplication is given by polynomials defined over the rational field $\boldsymbol{Q}$. Let $N_{\boldsymbol{Q}}(\boldsymbol{X})$ denote the rational points of $N$ relative to coordinate system. Now let $\left(y_{1}, \ldots, y_{n}\right)$ $=\boldsymbol{Y}$ be another coordinate system in $N$ such that multiplication in $N$ is given by polynomials defined over $\boldsymbol{Q}$. Let $N_{\boldsymbol{Q}}(\boldsymbol{Y})$ denote the rational points in $N$ relative to this polynomial rule of multiplication. Then it may happen that $N_{\boldsymbol{Q}}(\boldsymbol{X})$ and $N_{\boldsymbol{Q}}(\boldsymbol{Y})$ are not isomorphic. We will call $N_{\boldsymbol{Q}}(\boldsymbol{X})$ a rational form of $N$. Thus a nilpotent Lie group may have nonisomorphic rational forms (see $[\mathrm{S}, \mathrm{J}]$ for examples). However in our discussion we will always be dealing with a fixed rational form and so we shall write $N_{\boldsymbol{Q}}$ rather than $N_{\boldsymbol{Q}}(\boldsymbol{X})$. It should also be remarked that not every nilpotent Lie group has a rational form (see $[\mathrm{M}, \mathrm{A} ; \mathbf{1}]$ for example).

Let $N$ be a nilpotent Lie group and let $N_{\boldsymbol{Q}}$ be a rational form of $N$. Let $\pi$ be a subgroup of $N_{\boldsymbol{Q}}$. Then $\pi$ is discrete if and only if $\pi$ is finitely generated. It is easy to see that, if $\pi$ is discrete, it is finitely generated as this follows immediately from the fact that $\pi$ is the fundamental group of the compact manifold $A_{h}(\pi) / \pi$. Hence it is the converse that needs our attention.

By standard algebraic group arguments it is easy to see that it is no 
restriction to assume that $A_{h}(\pi)=N$. Let us now consider $\pi /[N, N] \cap \pi \subset$ $N_{\boldsymbol{Q}} /\left[N_{\boldsymbol{Q}}, N_{\boldsymbol{Q}}\right] . \quad$ It is finitely generated in the rational vector space $N_{\mathbf{Q}} /\left[N_{\mathbf{Q}}, N_{\mathbf{Q}}\right]$ and so $\pi /[N, N] \cap \pi$ is discrete in $N /[N, N]$ and, since $A_{h}(\pi)=N,(N /[N, N]) /(\pi /[N, N] \cap \pi)$ is compact. But, unlike general groups, subgroups of finitely generated nilpotent groups are finitely generated. Hence $\pi \cap\left[N_{\mathbf{Q}}, N_{\mathbf{Q}}\right]$ is finitely generated and so by induction $\pi \cap\left[N_{\mathbf{Q}}, N_{\mathbf{Q}}\right]$ is discrete in $[N, N]$. These facts combine to prove that $\pi$ is discrete in $N$.

By an argument similar to the one above, it can be shown that if $\pi_{1}$ and $\pi_{2}$ are finitely generated subgroups of $N_{\boldsymbol{Q}}$ such that $A_{h}\left(\pi_{1}\right)=A_{h}\left(\pi_{2}\right)$ then $\pi_{1}$ and $\pi_{2}$ are commensurable. (Recall that two groups $G_{1}$ and $G_{2}$ are called commensurable if $G_{1} \cap G_{2}$ is of finite index in each.)

Let us now prove one of the fundamental existence theorems in the theory of nilmanifolds. Let $N$ be a nilpotent Lie group with rational form $N_{\boldsymbol{Q}}$. Then there exists $\pi \subset N_{\mathbf{Q}}$ such that $\pi$ is a discrete subgroup of $N$ and $N / \pi$ is compact.

Let $M_{\boldsymbol{Q}} \subset N_{\mathbf{Q}}$ be of codimension 1 or such that $N_{\mathbf{Q}} / M_{\mathbf{Q}}$ is isomorphic to $Q$ and let the real points of $M_{Q}$ be $M$. By induction on the dimension of $N$ there exists $\Delta \subset M_{\boldsymbol{Q}}$ which satisfies the conclusion of our theorem relative to $M$. Now it is easily seen that $N_{\boldsymbol{Q}}=\boldsymbol{Q} \ltimes M_{\boldsymbol{Q}}$ where we will use the notation $A \ltimes B$ to denote the semidirect product of $A$ with the normal subgroup $B$. Now let $q \neq 0, q \in \boldsymbol{Q}$, and in $N_{\boldsymbol{Q}}$ consider the subgroup $\pi$ generated by $q$ and $\Delta$. Since $\Delta$ is finitely generated, so is $\pi$, and, since $A_{h}(\Delta)=M$, it is easily seen that $A_{h}(\pi)=N$. This proves our assertion.

Now let $L(N)$ denote as usual the Lie algebra to the nilpotent Lie group $N$ and let exp: $L(N) \rightarrow N$ denote the exponential mapping. Then by the Hausdorff-Campbell formula it follows that the exponential mapping is a homeomorphism. We will denote the inverse mapping to exp by log. A discrete subgroup $\pi \subset N$ will be called a lattice nilpotent group if $\log \pi$ is a subgroup of the vector space $L(N)$. Not every discrete subgroup $\pi$ of $N$ such that $A_{h}(\pi)=N$ is a lattice subgroup of $N$. For instance, if $N_{3}$ is given by

$$
N_{3}=\left\{\left(\begin{array}{lll}
1 & x & z \\
0 & 1 & y \\
0 & 0 & 1
\end{array}\right)\right\}, \quad x, y, z \in \boldsymbol{R}
$$

then

$$
\pi=\left\{\left(\begin{array}{ccc}
1 & n_{1} & n_{3} \\
0 & 1 & n_{2} \\
0 & 0 & 0
\end{array}\right)\right\}, \quad n_{i} \in \boldsymbol{Z}, i=1,2,3
$$


is not a lattice nilpotent group, but the group $\pi_{1}$ generated by $\pi$ and

$$
\left(\begin{array}{lll}
1 & 0 & \frac{1}{2} \\
& 1 & 0 \\
& & 1
\end{array}\right)
$$

is a lattice nilpotent group.

The definition and the basic facts about the existence of lattice nilpotent groups are due to $\mathrm{C}$. C. Moore $[\mathrm{M}, \mathrm{C}]$. Moore showed the following that will be of great importance to us in our later discussion. Let $\pi \subset N_{\boldsymbol{Q}} \subset N$ be discrete. Then there exists a lattice nilpotent subgroup $\Delta \supset \pi$ such that $\Delta / \pi$ is finite. We will not prove this. However the proof of the assertion below can be easily modified to prove this fact.

Let $\left(X_{1}, \ldots, X_{n}\right)$ be a basis of $L(N)$ such that $N_{\boldsymbol{Q}}=\exp \left(\sum q_{i} X_{i}\right), q_{i} \in \boldsymbol{Q}$, $i=1, \ldots, n$. Let $G$ be a completely reducible group of automorphisms of $L(N)$ contained in $G L(n, Z)$ relative to the basis $\left(X_{1}, \ldots, X_{n}\right)$. Then $N_{\boldsymbol{Q}}$ contains a lattice nilpotent subgroup $\Delta$ such that $A_{h}(\Delta)=N$ and $G$ maps $\Delta$ onto itself.

In $L(N)$ let $\mathscr{L}$ be the lattice generated by $X_{1}, \ldots, X_{n}$, i.e., $\mathscr{L}=\left\{\sum m_{i} X_{i} \mid m_{i}\right.$ $\in Z, i=1, \ldots, n\}$. Let $A$ be the last nontrivial term of the lower central series of $N$ and let $L(A) \cap \mathscr{L}=A_{X}$. Since $A$ is a rational subgroup of $N_{\boldsymbol{Q}}, A_{X}$ is a lattice subgroup of $A$ which we will identify with $L(A)$. Now consider $M_{Q}=N_{Q} / A_{Q}$. Since $G$ acts completely reducible, there is a rational vector subspace $W$ of $L(N)$ relative to $X_{1}, \ldots, X_{n}$, such that $L(N)=L(A) \oplus W$, and $W$ is $G$-invariant. Clearly $G$ maps $W \cap \mathscr{L}$. onto itself. If we identify $W$ and $L(M)$ under the natural 1-1 mappings we have that $\mathscr{L}^{*}=W \cap \mathscr{L}$ is a lattice invariant under $G$ acting as automorphisms of $M_{\mathbf{Q}}$. Hence by induction there is a lattice nilpotent subgroup $\Delta^{*}$ in $L(M)$ or $W$ which is invariant under $G$. Now let $\Delta$ be the subgroup of $N$ generated by $\exp (\delta), \delta \in \Delta^{*}$. It is easy to see that $\delta$ is a finitely generated subgroup of $N$ and for any element $\delta \in \Delta, \log \delta$ may be written uniquely as $a+\delta^{*}, a \in L(A)$ and $\delta^{*} \in \Delta^{*}$. Since $A$ is central it follows that the set of all points of $a$ that occur as the $A$ coordinate of $\log \delta, \delta \in \Delta$, is a subgroup $B$ of $A_{\boldsymbol{Q}}$ and is finitely generated. Hence, there exists $k \in Z^{+}$such that $A_{X} / k$ contains $B$. One now verifies that the group $B \Delta$ is a lattice nilpotent subgroup of $N_{\boldsymbol{Q}}$ invariant under $G$.

There is one further concept that is important about lattice nilpotent groups that we should mention before leaving this subject. This is the concept of a lattice hull and is defined as follows. Let $\pi \subset N_{\boldsymbol{Q}} \subset N$ be discrete. Then there exists a lattice nilpotent subgroup $\Delta \supset \pi$ such that $\Delta \subset N_{Q}$ and $\Delta / \pi$ is finite. If we let $\pi_{L}$ denote the intersection of all the lattice nilpotent subgroups that contain $\pi$ it is easy to see that $\pi_{L}$ is the 
smallest lattice nilpotent subgroup of $N_{\boldsymbol{Q}}$ containing $\pi$. We call $\pi_{L}$ the lattice hull of $\pi$.

4. Compact nilmanifolds. Let $\pi$ be a discrete subgroup of a nilpotent Lie group $N$ such that $\pi$ is cocompact in $N$ or equivalently $N / \pi$ is compact. Then $\pi$ uniquely determines $N$. This statement may be made precise as follows :

Let $\pi_{i} \subset N_{i}, i=1,2$, be discrete, cocompact subgroups of nilpotent Lie groups $N_{i}, i=1,2$. Further, let

$$
\alpha: \pi_{1} \rightarrow \pi_{2}
$$

be an isomorphism. Then $\alpha$ extends uniquely to an isomorphism

$$
\alpha^{*}: N_{1} \rightarrow N_{2} \text {. }
$$

We will prove this theorem by induction on the dimension of $N_{1}$. Let $M_{1}$ be a connected subgroup of $N_{1}$ of codimension 1 and such that $M_{1} / \pi_{1} \cap M_{1}$ is compact. (The existence of $M_{1}$ follows from the fact that the image of $\pi_{1}$ in $N_{1} /\left[N_{1}, N_{1}\right]$ is discrete and cocompact.) Let $\pi_{1}^{*}=\pi_{1} \cap M_{1}$ and note that $\pi_{1}=Z \ltimes \pi_{1}^{*}$ and $N_{1}=R_{1} \ltimes M_{1}$ with $Z_{1} \subset R_{1}$. Now let $\pi_{2}=\alpha\left(Z_{1}\right) \ltimes \alpha\left(\pi_{1}^{*}\right)$ and let $M_{2}=A_{h}\left(\alpha\left(\pi_{1}^{*}\right)\right) \subset N_{2}$. Then, by induction, $\alpha$ extends uniquely to an isomorphism $\alpha_{1}^{*}: M_{1} \rightarrow M_{2}$ since $\alpha\left(\pi_{1}^{*}\right)$ is discrete and cocompact in $M_{2}$ and $\operatorname{dim} M_{1}=\operatorname{dim} N_{1}-1$. Now it follows by a dimension argument that $N_{2}=\boldsymbol{R}_{2} \ltimes M_{2}$ with $\alpha\left(Z_{1}\right)=Z_{2} \subset \boldsymbol{R}_{2}$. We next note that $Z_{1}$ acts by inner automorphisms of $\pi_{1}$ as a group of automorphisms of $\pi_{1}^{*}$ and hence $Z_{1}$ may be considered to act as a group of automorphisms of $M_{1}$. Further $Z_{1}$ acts unipotently on $M_{1}$ and so the algebraic hull of $Z_{1}$ acting on $M_{1}$ is exactly the same as the action of $\boldsymbol{R}_{1}$ on $M_{1}$. A similar statement holds for $\alpha\left(Z_{1}\right)=Z_{2}$ acting on $M_{2}$. Now $\alpha: Z_{1} \rightarrow Z_{2}$ extends uniquely to $\alpha_{1}^{*}: \boldsymbol{R}_{1} \rightarrow \boldsymbol{R}_{2}$. We now define $\alpha^{*}: \boldsymbol{R}_{1} \ltimes M_{1} \rightarrow \boldsymbol{R}_{2} \ltimes M_{2}$ by $\alpha^{*}\left(r_{1} m_{1}\right)=\alpha_{1}^{*}\left(r_{1}\right) \alpha_{2}^{*}\left(m_{2}\right)$. It is now a straightforward verification that $\alpha^{*}$ is the desired unique isomorphism extending $\alpha$.

The type of argument we have just used can also be used to prove the following important fact. Let $\pi \subset N, N$ a nilpotent Lie group, be discrete and cocompact. Then there exists a rational form $N_{\boldsymbol{Q}}$ such that $\pi \subset N_{\mathbf{Q}}$.

Again the proof is by induction on the dimension of $N$ and we begin by choosing $M \subset N$ such that $M / \pi \cap M$ is compact, $N=\boldsymbol{R} \ltimes M, \pi \cap \boldsymbol{R}=\boldsymbol{Z}$ and $\pi=Z \ltimes \pi_{L}^{*}$ where $\pi^{*}=\pi \cap M$. By induction there exists a rational form $M_{Q}$ of $M$ such that $M_{Q} \supset \pi^{*}$. Let $\pi_{L}^{*}$ denote the lattice hull of $\pi^{*}$ and recall that $\boldsymbol{Z}$ acts as a group of automorphisms of $\pi_{L}^{*}$ and so we may view $Z$ as a subgroup $A$ of $G L(n, Z)$, where $n=\operatorname{dim} M$. Hence $A_{h}(A)_{Q}$ is a subgroup of $G L(n, Q)$ of dimension 1 and contained in $A(M)$. It is now easily seen that $N_{\boldsymbol{Q}}=A_{h}(A)_{\boldsymbol{Q}} \times M_{\boldsymbol{Q}}$ is a rational form of $N$.

We can repeat this type of argument once more to show that if $\pi$ is any 
finitely generated torsion free nilpotent group then there exists a nilpotent Lie group $N$ such that $\pi \subset N$ and $N / \pi$ is compact. The argument rests on the group theoretic fact that such groups $\pi$ have a semidirect product representation of the form $\pi=Z \ltimes \pi^{*}$, where $\pi^{*}$ is again torsion free, finitely generated and nilpotent. Thus there exists $M$ such that $M$ is a nilpotent Lie group and $\pi^{*} \subset M$ with $M / \pi^{*}$ compact. But then $Z$ acts as aroup of unipotent automorphisms of $M$ and so we may form $N=A_{h}(Z) \ltimes M$ and this group is easily seen to satisfy the conclusions of our theorem.

Since $N$ and $N_{Q}$ are uniquely attached to $\pi$ and if $\pi$ is faithfully imbedded in $U(m)$ for some $m, N$ will be isomorphic to the algebraic hull of $\pi$ in $U(m)$. We will often call $N$ the algebraic hull of $\pi$. We will often denote $N$ and $N_{\boldsymbol{Q}}$ by $\pi_{\boldsymbol{R}}$ and $\pi_{\boldsymbol{Q}}$, respectively.

We come now to our final result about compact nilmanifolds. Compact nilmanifolds with isomorphic fundamental groups are diffeomorphic. To see this, note that if $X_{1}$ and $X_{2}$ are compact nilmanifolds with fundamental groups $\pi_{1}$ and $\pi_{2}$, respectively, then there exist nilpotent Lie groups $N_{1}$ and $N_{2}$ such that $\pi_{i}$ is a discrete cocompact subgroup of $N_{i}, i=1,2$, and $X_{1} \approx N_{i} / \pi_{i}, i=1,2$. Now let $\alpha: \pi_{1} \rightarrow \pi_{2}$ be an isomorphism. Then $\alpha$ extends uniquely to an isomorphism $\alpha^{*}: N_{1} \rightarrow N_{2}$ and thus induces a diffeomorphism $\alpha^{* *}: N_{1} / \pi_{1} \rightarrow N_{2} / \pi_{2}$.

\section{Chapter III : Solvable LiE GROUPS AND SOlvMANIFOLdS}

1. Examples. Let $N$ be a nilpotent Lie group and let $\boldsymbol{R}^{\boldsymbol{s}}$ be the $s$-dimensional vector group. A (connected and simply connected) solvable Lie group is any Lie group $R$ satisfying the exact sequence

$$
1 \rightarrow N \rightarrow R \rightarrow R^{s} \rightarrow 1 .
$$

Now let $H$ be a closed subgroup of the solvable Lie group $R$. In this Chapter we will study the homogeneous spaces $R / H$ which are called solvmanifolds.

Let us begin our discussion by some examples that show that many of the assertions proven for nilpotent groups and nilmanifolds are false for solvmanifolds.

EXAmple 1. Let $\boldsymbol{C}$ denote the complex numbers and let $\boldsymbol{R}=\boldsymbol{R} \rtimes \boldsymbol{C}^{2}$, where $\boldsymbol{R}$ acts on $\boldsymbol{C}^{2}$ by

$$
\operatorname{ad}(t)\left(z_{1}, z_{2}\right)=\left(e^{2 \pi i t} z_{1}, e^{2 \pi i \lambda t} z_{2}\right), \quad \lambda \text { irrational, }
$$

$t \in \boldsymbol{R}$ and $\left(z_{1}, z_{2}\right) \in \boldsymbol{C}^{2}$. The solvable Lie group $\boldsymbol{R}$ is not an algebraic group in any matrix representation because the image of $R$ under the adjoint representation in $G L(5, R)$ is not a closed subset of $G L(5, R)$. If $R$ were an algebraic group in any representation then the image of $R$ under the adjoint representation would have to be an algebraic group and hence closed. 
EXAMPLE 2. The group $R_{2}$ of affine transformations of the line

$$
R_{2}=\left\{\left(\begin{array}{ll}
a & b \\
0 & 1
\end{array}\right)\right\}, \quad a, b \in \boldsymbol{R},
$$

has no discrete cocompact subgroup although in the above matrix representation it is a rational algebraic group.

EXAMPLE 3. The Klein bottle is a compact solvmanifold. Once we have shown this, we will have proven that not every compact solvmanifold can be written in the form $R / \Gamma$, where $\Gamma$ is a discrete subgroup of the solvable Lie group $R$. To see that the Klein bottle can be presented as a solvmanifold, let $\boldsymbol{R}=\boldsymbol{R} \ltimes \boldsymbol{C}$, where $\operatorname{ad}(t)(z)=e^{2 \pi i t} z, t \in \boldsymbol{R}, z \in \boldsymbol{C}$. If the elements of $R$ are given by $(t, z), t \in R$ and $z \in C$, let $H$ be the closed subgroup consisting of all elements of the form $(m / 2, n+i y), m, n \in \boldsymbol{Z}, y \in \boldsymbol{R}$. Then it is easily seen that $R / H$ is the Klein bottle.

EXAMPLE 4. Let $R$ be the universal covering group of the group of rigid motions of the plane. We may write this group as $R=R \ltimes C$ where $\operatorname{ad}(t) z=e^{2 \pi i t} z, t \in \boldsymbol{R}, z \in \boldsymbol{C}$; i.e., it is the same group as in Example 3. Let $\Gamma$ be the subgroup of $R$ consisting of $\left(m, n_{1}+i n_{2}\right), m, n_{1}, n_{2} \in \boldsymbol{Z}$, Then it is easily seen that $R / \Gamma$ is topologically the three-dimensional torus. Since $R$ is nonabelian, this shows that, for solvable Lie groups, isomorphisms of discrete cocompact subgroups may not extend to the connected groups containing them.

These examples show that the attack used in the study of nilmanifoldsa direct approach using algebraic groups - will not work for solvmanifolds. Although much work has been done in the study of solvmanifolds since the fundamental paper of Mostow in 1954 [M, G; 1], the global theory of solvmanifolds and the role played by algebraic groups in the study of solvmanifolds first became available in joint work of the author and $\mathbf{R}$. Tolimieri in 1970 [A, L; 8, 13]. It was in $[\mathrm{A}, \mathrm{L} ; 8]$ that we first established the fundamental global result - every solvmanifold is a vector bundle over a compact solvmanifold - a result first conjectured by Mostow in his 1954 paper. Before we begin the study of solvmanifolds let us set the stage by establishing how we will think about solvable Lie groups. The basic idea we will discuss can be traced back through the works of $\mathrm{H}$. C. Wang $[\mathrm{W}, \mathrm{H}]$ and A. I. Malcev $[\mathrm{M}, \mathrm{A} ; 2]$ and much further back into the history of group theory. It is interesting to note that the construction we are going to discuss and the wreath-product of groups have the same genesis.

2. The structure of solvable Lie groups-splitting theorems. There exist two fairly recent and complete accounts of the ideas in this section in $[\mathrm{A}, \mathrm{L} ; \mathbf{5}]$ and $[\mathrm{T}, \mathrm{R} ; \mathbf{1}]$. However, we will present an outline of this material that should help to serve as a guide through the above mentioned papers.

Let $R$ be a solvable Lie group. Then $R$ has a unique maximal normal 
nilpotent connected subgroup which is called the nil-radical of $R$ and which we will denote usually by $N$. Then $R$ satisfies the exact sequence

$$
1 \rightarrow N \rightarrow R \rightarrow R^{s} \rightarrow 1
$$

and so $N \supset[R, R]$. Thus the description of $R$ involves the "action" of $\boldsymbol{R}^{s}$ on $N$ and a group extension with nonabelian kernel. It is well known that it is very difficult to work with group extensions that have nonabelian kernels. It is also clear from Chapter II that we have great control over nilmanifolds because of the algebraic group structure of nilpotent Lie groups. Hence we want to describe solvable Lie groups in terms of groups that look as much like algebraic groups as possible. In order to do this, we will replace presenting $R$ as an exact sequence by presenting $R$ as a subgroup of a special sort of group.

Now if $R \subset G L(n, R)$ is an algebraic solvable Lie group, then $R=T \ltimes U$, where $U$ is a connected subgroup of $U(n)$ and $T$ is an abelian group of semisimple matrices. (Although $U$ is nilpotent, it is important to note that $U$ need not be the nil-radical of $R$.)

At this point two options are open to us. We can use a nonintrinsic approach that makes use of the Ado imbedding theorem or an intrinsic approach that is a modification of ideas first introduced by A. I. Malcev in $[\mathrm{M}, \mathrm{A} ; \mathbf{2}]$. We have chosen the second approach.

Let $L(R)$ denote as usual the Lie algebra of $R$ and consider the adjoint representation $\operatorname{ad}(R)$ on $L(R)$. If $A(L(R))$ denotes the algebraic group of automorphisms of $L(R)$, then $\operatorname{ad}(R) \subset A(L(R))$ and so $A_{h}(\operatorname{ad}(R))$, the algebraic hull of $\operatorname{ad}(R)$, is contained in $A(L(R))$. Since $\operatorname{ad}(R)$ is solvable, $A_{h}(\operatorname{ad}(R))$ is a solvable algebraic group and so

$$
A_{h}(\operatorname{ad}(R))=T \ltimes U
$$

where $T$ is semisimple abelian and $U$ is unipotent. In $T$ consider the image of $\operatorname{ad}(R)$ and denote this by $T_{R}$. We may view $T_{R}$ as a group of automorphisms of $R$ and so we may form

$$
R_{s}=T_{R} \ltimes R .
$$

If $R$ satisfies an exact sequence

$$
1 \rightarrow N \rightarrow R \rightarrow R^{s} \rightarrow 1
$$

where $N$ is the nil-radical of $R$, it is easy to see that $\operatorname{dim} T_{R}=s$ and if $\sim$ denotes universal covering group then

$$
R_{s}^{\tilde{N}}=T_{R}^{\tilde{R}} \ltimes R=R^{s} \ltimes R .
$$

We have called $R_{s}$ the semisimple splitting of $R$, although the name Malcev splitting might be also appropriate. Let us now list the basic properties of $R_{s}$. 
1. $T_{R}$ acts as an abelian semisimple group of automorphisms of $R$.

2. $R_{s}=M_{R} \rtimes T_{R}=R \rtimes T_{R}$, where $M_{R}$ is the nil-radical of $R_{s}$.

3. $R_{s}$ is uniquely determined by properties 1 and 2 .

We will often call $M_{R}$ the nil-shadow of $R$.

We may also relate $R_{s}^{\sim}$ to some exact sequences. To be precise $R_{s}^{\sim}$ satisfies the commutative diagram below where all horizontal sequences are exact and all vertical arrows are monomorphisms with the right hand arrow being an isomorphism.

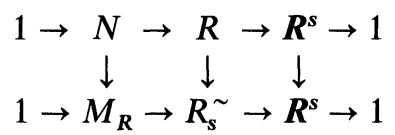

The crucial property is that the bottom exact sequence splits. Thus we see the reason for the name splitting in semisimple splitting.

One of the facts that makes $R_{s}$ so useful is that every automorphism $\theta$ of $R$ gives rise to an automorphism (not unique) of $R_{s}$. The basic tool for understanding this result is the following fundamental general theorem of Mostow [M, G ; 2] which we will call the "conjugacy theorem".

Let $G$ be a Lie group with a finite number of components and Lie algebra $L(G)$. Further, let $R$ be the radical of $G$ and $N$ the radical of [G, $G]$ (note, $N$ is nil-radical of $R$ ). Let $H_{1}$ and $H_{2}$ be two closed subgroups of $G$ each with the property that $\operatorname{ad}\left(H_{i}\right)$ is a maximal subgroup of $\operatorname{ad}(G)$ with respect to the property of semisimplicity; i.e., any invariant subspace of $L(G)$ under $\operatorname{ad}\left(H_{i}\right)$ has a complementary invariant subspace under $\operatorname{ad}\left(H_{i}\right), i=1,2$. Then there exists $n \in N$ such that $n H_{1} n^{-1}=H_{2}$.

The Mostow-conjugacy theorem enables us to prove (see $[\mathrm{A}, \mathrm{L} ; 5])$ the following: Let $R$ be a solvable Lie group with semisimple splitting $R_{s}$ and let $A(R)$ denote the group of automorphisms of $R$ and $A\left(R_{s}, R\right)$ the group of automorphisms of $R_{s}$ that map $R$ onto itself. Then the restriction mapping

$$
r: A\left(R_{s}, R\right) \rightarrow A(R)
$$

is a surjection and there is a monomorphism $c: A(R) \rightarrow A\left(R_{s}, R\right)$ such that $r \circ c$ is the identity on $A(R)$. Hence every group of automorphisms of $R$ lifts to $R_{s}$, but not uniquely.

3. A splitting theorem and a global theorem about solvmanifolds. Now just as Malcev proved that solvable Lie groups have semisimple splittings, H. C. Wang proved that certain disconnected solvable groups have splittings. For later purposes we will state Wang's results in a slightly more general form than in his paper [W, H] but in slightly less generality than the results established by Toliemeri in $[\mathrm{T}, \mathrm{R} ; \mathbf{1}]$.

By a CN group we will mean any closed subgroup of a nilpotent Lie group. Let $N$ be a nilpotent Lie group and let $H$ be a closed subgroup of 
$N$ with algebraic hull $A_{h}(H)$. Since $A_{h}(H)$ is the same no matter what nilpotent Lie group we take to contain $H$ as closed subgroup, we will adapt the notation $H_{R}=A_{h}(H)$. If $H_{0}$ is the identity component of $H$ then $H_{0}$ is normal in $H_{R}$ and $H_{R} / H_{0}$ contains a unique rational form, say $Q$, such that $Q \supset H / H_{0}$. Let $H_{Q}$ be the pre-image of $Q$ in $H_{R}$.

We will call a group a CS group if it is a closed subgroup of a solvable Lie group. One can demonstrate (see $[\mathrm{A}, \mathrm{L} ; \mathbf{1 4}$, Corollary 2]) that a CS group $D$ satisfies an exact sequence

$$
1 \rightarrow H \rightarrow D \rightarrow Z^{s} \oplus R^{t} \rightarrow 1
$$

where $H$ is a $\mathrm{CN}$ group. By the unique extension of automorphisms for $\mathrm{CN}$ groups, we have easily the following commutative diagram with horizontal exact sequences and vertical monomorphisms:

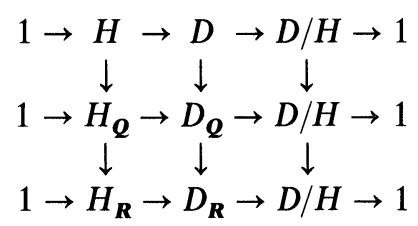

All the statements we are about to make regarding $D_{\boldsymbol{R}}$ also hold for $D_{\boldsymbol{Q}}$.

We will now show how to construct a semisimple splitting or simply a splitting of $D_{R}$. The method we will follow here is analogous to using the Ado theorem to prove the Malcev or semisimple splitting for solvable Lie groups. (The reader should note that in general $D_{R}$ has an infinite number of components.) What $\mathrm{H}$. C. Wang actually proved we will discuss in the next section and call it the abstract or the Wang splitting.

Let us begin by noting that if $D \subset R$ then $D_{R} \subset R$. Now let $R_{s}$ be the semisimple splitting at $R$ and let $M_{R}$ be the nil-shadow of $R$. Further let $R_{s} / M_{R}=T_{R}$ and let the image of $D_{R}$ in $T_{R}$ be $T^{*}$. Our main assertion is that there exists a monomorphism $\alpha: T_{R} \rightarrow A(R)$ such that $R_{s}=M_{R} \rtimes \alpha\left(T_{R}\right)$ and $\alpha\left(T^{*}\right)$ maps $D_{R}$ onto itself.

Since we wish to be able to use algebraic group techniques to prove our assertion, we will begin by taking a particular faithful matrix representation of $R_{s}$. To be more precise, we will use the Birkhoff Imbedding Theorem which states that if $N$ is a nilpotent Lie group with automorphism group $A(N)$ then there is a faithful imbedding $B: N \rightarrow U(n)$ and $B: A(N) \rightarrow G L(n, R)$ such that $B(\theta(n))=B(\theta) B(n) B(\theta)^{-1}$ where $\theta \in A(N)$ and $n \in N$. This representation of $N \rtimes A(N)$ has the property that if $R_{s}=T_{R} \ltimes M_{R}$ then not only is $B\left(M_{R}\right)$ unipotent, but $B\left(T_{R}\right)$ is semisimple.

We may now proceed to prove our assertion. Let $A=A_{h}\left(B\left(D_{R}\right)\right)$. Then $[A, A]$ is the algebraic hull of $\left[B\left(D_{R}\right), B\left(D_{R}\right)\right]$. But $\left[B\left(D_{R}\right), B\left(D_{R}\right)\right] \subset B\left(H_{R}\right)$, which, being a connected nilpotent group, is algebraic. Hence 
$[A, A] \subset B\left(H_{R}\right)$. Now let $A=M_{A} \rtimes T_{A}$, where $M_{A}$ is unipotent and $T_{A}$ is semisimple. Now let $T_{D}^{*}$ be the image of $B\left(D_{R}\right)$ in $T_{A}$. Then, for $c \in B\left(D_{R}\right)$ and $t \in T_{A}$, we have

$$
[t, c]=t c t^{-1} c^{-1} \in B\left(H_{R}\right) \subset B\left(D_{R}\right) .
$$

Hence $t c t^{-1} \in B\left(D_{\mathbf{R}}\right)$. Now let $B\left(M_{\mathbf{R}}\right) \rtimes B\left(T_{\mathbf{R}}\right)$ be a semidirect product presentation of $B\left(R_{s}\right)$ such that

$$
B\left(T_{R}\right) \supset T_{A} \cap B\left(T_{D}^{*}\right) .
$$

Since $B$ is an isomorphism, this proves our assertion.

If we let $T_{D}$ denote the restriction of $T_{D}^{*}$ to $D_{R}$, we may form $\left(D_{R}\right)_{s}=$ $D_{R} \rtimes T_{D}$. We will call $\left(D_{R}\right)_{s}$ a semisimple or Wang splitting of $D_{R}$.

We may now argue as in $[\mathrm{A}, \mathrm{L} ; 5]$ and show that the following four conditions are satisfied.

1. $\left(D_{R}\right)_{s}$ has a maximal nilpotent normal subgroup, which we will call the nil-shadow of $D_{R}$ and denote by $H_{D} . H_{D}$ is a CN group. Let the algebraic hull of $H_{D}$ be denoted by $M_{D}$ and be called the real nil-shadow of $D$. The group $\left(H_{D}\right)_{Q}$ will be called the rational nil-shadow of $D$.

2. $\left(D_{R}\right)_{s}=H_{D} \ltimes T_{D}$.

3. The set-theoretic projection $p: H_{D} \ltimes T_{D} \rightarrow H_{D}$ maps $D_{R}$ homeomorphically onto $H_{D}$.

4. $D_{R}$ and $H_{D}$ generate $\left(D_{R}\right)_{s}$.

We will now use the above properties of splittings of CS groups to show that every solvmanifold is a vector bundle over a compact manifold. We will, in the next sections, introduce the abstract splitting theorem and indicate how these can be used to prove that every solvmanifold is a vector bundle over a compact solvmanifold and that compact solvmanifolds with isomorphic fundamental groups are homeomorphic.

Let $R$ be a solvable Lie group and let $D$ be a closed subgroup of $R$. Further, let $R_{s}=M_{R} \rtimes T_{R}$ be the semisimple splitting of $R$. We define the affine action of $R$ on $M_{R}$ as follows: Let $r \in R$ and let $r=(m, t)$, where $m \in M_{R}$ and $t \in T_{R}$. Let $m_{0} \in M_{R}$. We define $a(g) m_{0}=m t\left(m_{0}\right)$, where $t\left(m_{0}\right)$ is the image of $m_{0}$ under the automorphism determined by $t$. This defines $R$ as a group of transformations acting on $M_{R}$. Note that if we denote the left multiplication by $r$ in $R$ by $l_{r}$ and projection of $R$ onto $M_{R}$ by $p$, then

$$
p l_{r}=a(r) p .
$$

As an immediate corollary of this observation, we have that the solvmanifold $R / D$ is homeomorphic to $M_{R} / a(D)$, where $M_{R} / a(D)$ denotes the quotient space of $M_{R}$ under the affine action of $D$. This observation has the important consequence that the topological structure of $R / D$ depends on $M_{R}$ and $T_{D}^{*}$, but not on $T_{R}$. (This explains why nonuniqueness in the 
presentation of solvmanifolds is possible as exhibited in Example 4 of $\$ 1$ of this chapter.)

Now let $D$ be a CS group and let

$$
\left(D_{R}\right)_{s}=H_{D} \rtimes T_{D}
$$

be the semisimple splitting of $D_{R}$. Then if $D_{0}$ denotes the identity component of $D$, it can be verified that the nil-shadow of $D_{0}$ in $H_{D}$, which we will denote by $H_{0}$, is normal in $\left(D_{R}\right)_{s}$. Let $T_{D}^{1}$ denote the image of $T_{D}$ on $H_{D} / H_{0}$. Then we easily have that

$$
\left(\left(D / D_{0}\right)_{R}\right)_{s}=\left(H_{D} / H_{0}\right) \rtimes T_{D}^{1} ;
$$

thus we have established that if $D$ is a CS group then the orbit of $D$ acting affinely on its real nil-shadow $M_{D}$ is compact and has fundamental group $\Gamma=D / D_{0}$, where $\Gamma$ satisfies the exact sequence

$$
1 \rightarrow \pi \rightarrow \Gamma \rightarrow Z^{s} \rightarrow 1
$$

where $\pi$ is the fundamental group of a compact nilmanifold or, equivalently, a finitely generated torsion free nilpotent group. A group $\Gamma$ satisfying the above conditions will be called a FS group.

Now let $D$ be a closed subgroup of $R$ and let the semisimple splitting of $R$, $R_{s}=M_{R} \rtimes T_{R}$ be chosen so that if $\left(D_{R}\right)_{s}=H_{D} \rtimes T_{D}^{*}$ then $H_{D} \subset M_{R}$ and $T_{D}^{*} \subset T_{R}$. Then $M_{D}$ the real nil-shadow of $D_{R}$ is a connected subgroup of $M_{R}$ which is invariant under $\left(D_{R}\right)_{s}$ and by the above discussion $M_{D} / a(D)$ is a compact manifold. Since $M_{R}$ is nilpotent, we will, for the rest of this discussion, view $M_{R}$ and $L\left(M_{R}\right)$ as identified by means of the exponential mapping. Let $V$ be a vector subspace of $M_{R}$ such that $T_{D}^{*}(V)=V$ and $M_{R}=M_{D} \oplus V$. Then one can verify that every element of $M_{R}$ can be written uniquely as $\delta v, \delta \in M_{D}$ and $v \in V$. Hence if $d=(m, t) \in D$, we have

$$
\operatorname{ad}(d)(\delta v)=m t(\delta) t(v)=m^{1} v^{1}
$$

where $m^{1} \in M_{D}$ and $v^{1} \in V$. Thus we see that the images of the sets $\delta V$, $\delta \in M_{D}$, gives a fiber bundle structure to $R / C \approx M_{R} / a(D)$ with $M_{D} / a(D)$ as base space and $V$ as fiber with the linear action of the abelian structure group $T_{D}^{*}$. This proves our first structure theorem. Every solvmanifold is a vector bundle over a compact manifold.

4. Abstract splitting theorems and uniqueness of compact solvmanifolds. In $\$ 3$ we established our first structure theorem for solvmanifolds. In the next two sections, we will establish two further properties : First, compact solvmanifolds are homeomorphic if and only if their fundamental groups are isomorphic; second, given any FS group it is the fundamental group of a compact solvmanifold.

Both of these results start from the Wang splitting theorem. Indeed, 
the first result is an almost immediate corollary of the Wang result while the second uses the Wang splitting as a jumping off point. We will therefore begin this section with a discussion of the Wang splitting.

In $\$ 3$ we saw that if $D$ is a closed subgroup of a solvable Lie group $R$, or what we have been calling a CS group, then $D$ satisfies an exact sequence

$$
1 \rightarrow H \rightarrow D \rightarrow Z^{s} \oplus R^{t} \rightarrow 1
$$

where $H$ is a $\mathrm{CN}$ group. The converse is false. In other words there exist groups $D$ satisfying (1) which cannot be imbedded as closed subgroups of connected, simply connected solvable Lie groups. However, H. C. Wang proved in $[\mathrm{W}, \mathrm{H}]$ that if $D$ satisfies (1) then $D_{\boldsymbol{R}}$ and $D_{\mathbf{Q}}$ have semisimple splittings $\left(D_{\mathbf{R}}\right)_{s}$ and $\left(D_{\mathbf{Q}}\right)_{s}$ as defined for CS groups. One may then use the Birkhoff imbedding theorem to show that groups satisfying (1) have faithful matrix representations and can be imbedded in simply connected solvable Lie groups with a finite number of components. (Note that if we knew for some reason in advance that a group satisfying (1) had a faithful matrix representation, the proof of its having a Wang splitting would be a simple matter.)

Let $D$ be a group satisfying the exact sequence (1). Again we will state all our results for the group $D_{\mathbf{R}}$ with the understanding that exactly the same results hold for $D_{\boldsymbol{Q}}$. Let $A\left(D_{\boldsymbol{R}}, H_{\boldsymbol{R}}\right)$ denote all continuous automorphisms of $D_{R}$ that map $H_{R}$ onto itself and induce the trivial automorphism of $D_{\mathbf{R}} / H_{\mathbf{R}}$. We will say that $\theta \in A\left(D_{\mathbf{R}}, H_{\mathbf{R}}\right)$ is unipotent if $\theta$ induces a unipotent automorphism of $H_{\boldsymbol{R}}$. We will call $\theta \in A\left(D_{\boldsymbol{R}}, H_{\boldsymbol{R}}\right)$ semisimple if

(a) there exists a cross section $f: \boldsymbol{Z}^{s} \oplus \boldsymbol{R}^{t} \rightarrow D_{\boldsymbol{R}}$ such that $\theta f(z)=f(z)$, $z \in \boldsymbol{Z}^{s} \oplus \boldsymbol{R}^{t}$.

(b) $\theta \mid H_{R}$ is semisimple.

Wang has shown that, just as for linear transformations, every $\theta \in A\left(D_{\boldsymbol{R}}, H_{\boldsymbol{R}}\right)$ can be written uniquely as a product of two commuting automorphisms $s(\theta)$ and $n(\theta)$ both in $A\left(D_{\mathbf{R}}, H_{\mathbf{R}}\right)$ such that $s(\theta)$ is semisimple and $n(\theta)$ is unipotent.

Now let $\operatorname{ad}\left(D_{R}\right)$ denote the subgroup of $A\left(D_{R}, H_{R}\right)$ consisting of inner automorphisms. Let $\left(D_{R}\right)_{w}$ be the subgroup of $A\left(D_{R}, H_{R}\right)$ generated by all the semisimple and unipotent parts of $\theta \in \operatorname{ad}\left(D_{R}\right)$. Let $B_{D}$ denote a maximal abelian subgroup of $\left(D_{\mathbf{R}}\right)_{w}$ consisting of semisimple matrices. Then H. C. Wang has shown the following:

1. There exists a cross section $f: \boldsymbol{Z}^{s} \oplus \boldsymbol{R}^{t} \rightarrow D_{R}$ such that for each $z \in \boldsymbol{Z}^{s} \oplus \boldsymbol{R}^{t}$ we can find $b \in B_{D}$ such that $\operatorname{ad}(f(z)) b^{-1}$ is unipotent.

2. The group $U_{D}$ generated by the unipotent elements $\operatorname{ad}(f(z)) b^{-1}$ and $H_{\boldsymbol{R}}$ is nilpotent and a $\mathrm{CN}$ group which is such that

$$
1 \rightarrow H_{R} \rightarrow\left(U_{D}\right)_{R} \rightarrow Z^{s} \otimes R \oplus R^{t} \rightarrow 1 .
$$


3. $B_{D}$ acts as an abelian group of semisimple automorphisms on $\left(U_{D}\right)_{R}$ which maps $H_{R}$ onto itself and is trivial on $\left(U_{D}\right)_{R} / H_{R}$.

4. $\boldsymbol{Z}^{s} \otimes \boldsymbol{R} \oplus \boldsymbol{R}^{t}$ contains a lattice $Y$ such that if $f$ is a cross section of $Z^{s} \oplus \boldsymbol{R}^{t} \rightarrow D_{\boldsymbol{R}}$ satisfying 1 then there exists a cross section $g: Y \rightarrow\left(U_{D}\right)_{R}$ such that the unipotent parts of $\operatorname{ad}(f(y))$ equals $\operatorname{ad}(g(y))$ restricted to $H_{\boldsymbol{R}}$.

5. The group $\left(D_{R}\right)_{w}=U_{D} \rtimes B_{D}$.

Now let $D$ be a CS group. Apparently the definition of semisimple splitting of $D_{R}$ given in $\S 3$ depended on the choice of enveloping group $R$. We will now see that this is not the case. Let $\left(D_{R}\right)_{S}=M_{D} \rtimes T_{D}$ be a semisimple splitting of $D$ and let $\left(D_{R}\right)_{w}=U_{D} \rtimes B_{D}$. Then we may choose a semidirect product representation of $\left(D_{R}\right)_{w}$ such that $T_{D}=B_{D}$ as a group of automorphisms of $D_{R}$ and if $\beta$ assigns to each $m \in M_{D}$ the automorphism of $D_{\mathbf{R}}$ obtained by $m D_{\mathbf{R}} m^{-1}$ then

$$
\beta: M_{D} \rightarrow U_{D}
$$

is an epimorphism.

Now let $\xi$ be an automorphism of $D_{\boldsymbol{R}}$. Then $\xi$ induces an automorphism $\xi^{*}$ of $\left(D_{R}\right)_{w}$ if we define $\xi^{*}$ by

$$
\xi^{*}(h)=\xi h \xi^{-1}, \quad h \in\left(D_{R}\right)_{w} \subset A\left(D_{R}, H_{R}\right) .
$$

Now $\xi^{*}\left(B_{D}\right)$ acts semisimply on $U_{D}$ and so by the Mostow conjugacy theorem there exists $u \in U_{D}$ such that

$$
u B_{D} u^{-1}=\xi^{*}\left(B_{D}\right)
$$

where $u$ is in the range of $\left(B_{D}-I\right)$, where $I$ is the identity mapping. Note that $H_{R}$ maps onto the range of $\left(B_{D}-I\right)$. Hence there exists $n \in H_{R}$ such that

$$
\xi B_{D} \xi^{-1}=n B_{D} n^{-1} .
$$

This discussion may be formalized as follows: Let $D_{R}$ be given with semisimple splitting $\left(D_{R}\right)_{s}=H_{D} \rtimes T_{D}=D_{R} \rtimes T_{D}$. Let $\xi$ be an automorphism of $D_{\boldsymbol{R}}$. Then

(a) $\xi T_{D} \xi^{-1}=n T_{D} n^{-1}$ for $n \in H_{R}$,

(b) $\xi^{*}:\left(D_{\mathrm{R}}\right)_{s} \rightarrow\left(D_{\mathrm{R}}\right)_{s}$ defined by

$$
\xi^{*}(c, t)=\left(\xi(G), \xi t \xi^{-1}\right)
$$

is an automorphism extending $\xi$. This proves that groups $D_{R}$ have unique semisimple splittings and nil-shadows $H_{D}$. Since $D_{R}$ is a subgroup of $\left(D_{R}\right)_{s}$ that maps onto $T_{D}$ by the homomorphism with kernel $M_{D}$, it follows that we may view $T_{D}^{\tilde{D}}=D_{R} / H_{R}$ as a group of automorphisms of $H_{D}$ and form the group $\left(D_{R}\right)_{s}^{\sim}=H_{D} \rtimes T_{D}^{\sim}$.

These algebraic facts have the following immediate implications for solvmanifolds. Let $R_{i} / D_{i}$ be solvmanifolds that are vector bundles over 
the compact manifolds $M_{D_{i}} / a\left(D_{i}\right)$. Then if $D_{i} /\left(D_{i}\right)_{0}, i=1,2$, are isomorphic, the manifolds $M_{D_{i}} / a\left(D_{i}\right)$ are homeomorphic. This is an easy consequence of the algebraic uniqueness theorems established above and immediately implies that compact solvmanifolds with isomorphic fundamental groups are homeomorphic.

5. Existence of solvmanifolds. We have used the Wang splitting to show that if $D_{1}$ and $D_{2}$ are CS groups such that $D_{1} /\left(D_{1}\right)_{0}$ is isomorphic to $D_{2} /\left(D_{2}\right)_{0}$ then

$$
M_{D_{1}} / a\left(D_{1}\right) \approx M_{D_{2}} / a\left(D_{2}\right)
$$

and the fundamental group $\Gamma$ of $M_{D_{1}} / a\left(D_{1}\right), \Gamma=D_{1} /\left(D_{1}\right)_{0}$, satisfies the exact sequence

$$
1 \rightarrow \pi \rightarrow \Gamma \rightarrow Z^{s} \rightarrow 1
$$

where $\pi$ is a FN group. In this section we will show that given any group $\Gamma$ satisfying (1) there is a compact solvmanifold $X$ such that $\pi_{1}(X)=\Gamma$. This, combined with the results in $\$ 4$, will complete the proof that any solvmanifold is a vector bundle over a compact solvmanifold. We will not present the original proof here $[\mathrm{A}, \mathrm{L} ; 6]$, but will present a new proof that is both more invariant and gets deeper into the structure of solvmanifolds. We will begin by motivating the proof and explaining where the difficulties lie. The interested reader may compare this to the results and proofs in $[\mathrm{A}, \mathrm{L} ; \mathbf{1 6}]$.

We will begin by considering the special case where $\pi$ is abelian, say $Z^{t}$. Thus $\Delta$ satisfies the exact sequence

$$
1 \rightarrow Z^{t} \rightarrow \Delta \rightarrow Z^{s} \rightarrow 1 .
$$

In this special case we may proceed as follows to show why $\Delta$ is the fundamental group of a compact solvmanifold. First consider the commutative diagram, where horizontal sequences are exact and vertical arrows are monomorphisms :

$$
\begin{aligned}
& 1 \rightarrow \quad Z^{t} \rightarrow \Delta \rightarrow Z^{s} \rightarrow 1 \\
& 1 \rightarrow Z^{t} \stackrel{\downarrow}{\otimes} \underset{\Delta_{R}}{\otimes} \rightarrow \stackrel{\downarrow}{Z^{s} \rightarrow 1}
\end{aligned}
$$

Since $\boldsymbol{Z}^{t} \otimes \boldsymbol{R}=\boldsymbol{R}^{t}$, we have a natural imbedding $j$ of $\boldsymbol{Z}^{s}$ in $G L(t, \boldsymbol{R})$. What we would like to do is to complete the above diagram to the following:

$$
\begin{aligned}
& 1 \rightarrow Z^{t} \quad \rightarrow \Delta \rightarrow Z^{s} \quad \rightarrow 1
\end{aligned}
$$

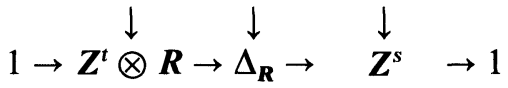

$$
\begin{aligned}
& 1 \rightarrow Z^{t} \otimes R \rightarrow \stackrel{\downarrow}{\otimes} \rightarrow Z^{s} \otimes R \rightarrow 1
\end{aligned}
$$


If this could be done we would easily have that $R / \Delta$ would be a compact solvmanifold with fundamental group $\Delta$. But the existence of the last exact sequence would imply that $j\left(\boldsymbol{Z}^{s}\right) \subset G L(t, \boldsymbol{R})$ is contained in a connected abelian subgroup of $G L(t, \boldsymbol{R})$. Since $G L(t, \boldsymbol{R})$ is not connected, it is easy to find $\Delta$ for which $R$ satisfying (4) does not exist.

However we know that if we consider $\boldsymbol{Z}^{t} \otimes \boldsymbol{C}, \boldsymbol{C}$ the complex numbers, instead of $\boldsymbol{Z}^{t} \otimes R$, we have $j\left(\boldsymbol{Z}^{s}\right) \subset G L(t, C)$ and now it is well known that we can always extend the homomorphism $j$ from $Z^{s}$ to $Z^{s} \otimes R$. One can now show, and we will see how in a few moments, to complete the commutative diagram below with the usual conventions in vertical and horizontal mappings.

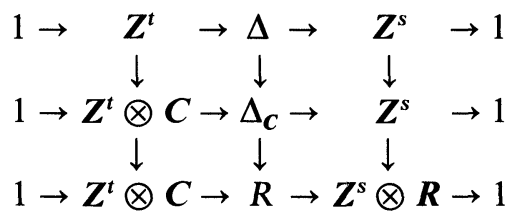

Unfortunately, $R / \Delta$ is not compact. This difficulty can be overcome by letting $D$ be the subgroup of $R$ generated by the image of $\Delta$ and the subgroup $\mathscr{I}$ of $\boldsymbol{Z}^{\mathrm{c}} \otimes C$ consisting of the purely imaginary vectors. It is easily checked that $D$ is closed in $R$ and that $\pi_{1}(R / D)=D / D_{0}=D / \mathscr{I}=\Delta$. Thus we see how to go about solving our problem in this special case.

Let us now see what happens when we try to do this in the general case of the exact sequence (1). Let $N$ be the algebraic hull of $\pi$ and let $N_{C}$ be the complex points of $N$. Then we may try to form (5) with $Z^{t} \otimes C$ replaced by $N_{\boldsymbol{c}}$. Unfortunately this attack does not work. One reason is that the subset of $N_{C}$ with purely imaginary coordinates does not form a subgroup of $N_{C}$ when $N$ is not abelian as the simplest examples will show.

Until now we have glossed over the problem of extending the cocycles that are implicit in the construction of diagrams (4) and (5). The reason for this was twofold: first to isolate the automorphism extension problem and second because the Wang splitting will enable us to overcome this difficulty. Let us now see how this is done.

Consider $\left(\Delta_{R}\right)_{s}^{\tilde{s}}=Z^{s} \ltimes H_{\Delta}$ and let $M_{\Delta}$ be the algebraic hull of $H_{\Delta}$. Then $M_{\Delta}$ satisfies the exact sequence

$$
1 \rightarrow Z^{s} \otimes R \rightarrow M_{\Delta} \rightarrow Z^{t} \otimes R \rightarrow 1 .
$$

Further we may form $\left(M_{\Delta}\right)_{c}$ by requiring it to satisfy the above exact sequence with $\boldsymbol{Z} \otimes \boldsymbol{R}$ replaced by $\boldsymbol{Z} \otimes \boldsymbol{C}$.

Now let $j\left(\boldsymbol{Z}^{t}\right) \subset G L(s, \boldsymbol{C})$ and let $j^{*}\left(\boldsymbol{Z}^{t} \otimes R\right)$ be an extension of $j$. Let $A$ be the algebraic hull of $j^{*}\left(Z^{t} \otimes R\right)$ and let $A=T_{A} \oplus U_{A}$ where $U_{A}$ is the unipotent part and $T_{A}$ the semisimple part of $A$. Let $u^{\prime}: A \rightarrow U_{A}$ have 
kernel $T_{A}$ and $s^{\prime}: A \rightarrow T_{A}$ have kernel $U_{A}$. Then form $u:\left(\boldsymbol{Z}^{t} \otimes R\right)$ $\rightarrow G L(s, \boldsymbol{C})$ and $s: \boldsymbol{Z}^{t} \otimes R \rightarrow G L(s, \boldsymbol{C})$ by $u=u^{\prime} \circ j^{*}$ and $s=s^{\prime} \circ j^{*}$. It should be remarked that $u$ is independent of how $j$ is extended to $j^{*}$, but $s$ is not. It is also easily seen how to form $\left(Z^{t} \otimes R\right) s^{*} \times\left(M_{\Delta}\right)_{c}$, where $\left(\boldsymbol{Z}^{t} \otimes R\right) s^{*}$ means that $\boldsymbol{Z}^{t} \otimes \boldsymbol{R}$ acts semisimply on $\left(M_{\Delta}\right)_{\boldsymbol{c}}$ so as to agree with $s$ when restricted to $Z^{s} \otimes C$ and to act trivially on $\left(M_{\Delta}\right)_{c} / Z^{s} \otimes C$. Further the following commutative diagram exists where $\delta$ is the mapping defined by $\delta(z)=s^{*}(z \otimes 1) \oplus(z \otimes 1), z \in \boldsymbol{Z}^{t}$, and all vertical arrows are monomorphisms:

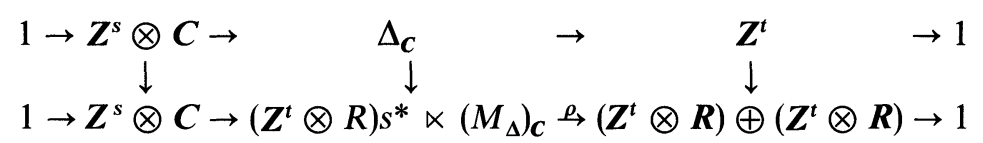

If we let $R=\rho^{-1}(z \otimes r \oplus z \otimes r), z \in Z^{t}, r \in \boldsymbol{R}$, it is easily seen that $R \subset\left(Z^{t} \otimes R\right) s^{*} \propto\left(M_{\Delta}\right)_{c}$ satisfies $(5)$.

Let us now go through the general arguments. We begin our discussion with the introduction of the concept of the free nilpotent Lie group of step $k$ generated by a vector space $V$. This will be our replacement for $Z^{s} \otimes C$ in the above argument. We will begin by recalling some of the basic properties of the free Lie algebra generated by $F(V)$ a vector space $V$.

1. Let $\alpha: V \rightarrow L$ be a linear mapping of $V$ into a Lie algebra $L$. Then $\alpha$ extends uniquely to an algebra homomorphism $\alpha^{*}: F(V) \rightarrow L$.

2. Let $G$ be a group of automorphisms of $V$. Then $G$ extends uniquely to a group of automorphisms of $F(V)$.

3. Let $G$ be a group of automorphisms of $V$ and assume that $W \subset V$ is a linear subspace that is $G$-invariant. Let $\mathscr{I}(W)$ be the ideal in $F(V)$ generated by $W$. Then $\mathscr{I}(W)$ is $G$-invariant.

4. Let $F_{k}(V)$ be the $k$ th term of the lower central series of $F(V)$. We call $F(V) / F_{k+1}(V)=F^{k}(V)$ the $k$ step free nilpotent Lie algebra generated by $V$. If $N$ is a $k$ step nilpotent Lie algebra and $\alpha: F(V) \rightarrow N$ is a homomorphism then $\alpha$ factors through $F^{k}(V)$.

Henceforth we will identify a nilpotent Lie algebra with the corresponding Lie group by means of the exponential mapping implicitly whenever this is convenient.

Before we proceed to a proof of our main assertion, let us pause to consider the following problem: Let $M$ be a connected simply connected nilpotent Lie group and let $\rho: \boldsymbol{R}^{s} \rightarrow A(M)$ be a homomorphism whose image is semisimple. Let $G=\left(\boldsymbol{R}^{s}\right) \rho \ltimes M$, where $\left(\boldsymbol{R}^{s}\right) \rho$ means that $\boldsymbol{R}^{s}$ acts on $M$ as the image of the mapping $\rho$.

Problem 1 . Give a necessary and sufficient condition for there to exist a solvable Lie group $R$ such that $R_{s}^{\sim}=G$.

Clearly if $M \rtimes\left(R^{s}\right) \rho$ is to be the $R_{s}^{\sim}$ for some $R$, there must exist a 
connected subgroup $N \subset M$, such that $N \supset[M, M]$ and

1. $N$ is $\rho\left(\boldsymbol{R}^{s}\right)$ invariant and $\rho\left(\boldsymbol{R}^{s}\right)$ acts trivially on $M / N$,

2. $\operatorname{dim} M-\operatorname{dim} N=s$.

Now assume that $G$ satisfies (1) and (2). Then $G / N=M / N \oplus R^{s}$. Let $\alpha: M / N \rightarrow R^{s}$ be a linear isomorphism and let $\Delta=\{(x, \alpha x) \mid x \in M N\}$. If $\mu: G \rightarrow G / N$ is the canonical map with kernel $N$, let $\mu^{-1}(\Delta)=R$. Then it is trivial to verify that $R_{s}=M \rtimes\left(R^{s}\right) \rho$.

We now want to look at a slight variant of this problem. Consider a group

$$
1 \rightarrow H \rightarrow D \rightarrow Z^{s} \rightarrow 1
$$

where $H$ is a CN group. Let $\left(D_{R}\right)_{s}^{\sim}=H_{D} \rtimes Z^{s}$ is the Wang splitting and let $M_{D}$ be the algebraic hull of $H_{D}$. Assume $\left(D_{R}\right)_{s}^{\sim} \subset M_{D} \rtimes\left(R^{s}\right) \rho$, where $\left(R^{s}\right) \rho$ acts semisimply on $M_{D}$.

Problem 2. Can we find $R \subset M_{D} \rtimes\left(R^{s}\right) \rho$ such that $R \supset D$ and $R_{s}^{\sim}=M_{D}$ $\rtimes\left(\boldsymbol{R}^{s}\right) \rho$.

The answer is of course yes and is proven by combining the last two arguments.

Now let $\Gamma$ satisfy the exact sequence

$$
1 \rightarrow \pi \rightarrow \Gamma \rightarrow Z^{s} \rightarrow 1
$$

and let $\left(\Gamma_{R}\right)_{s}^{\tilde{}}=H_{\Gamma} \rtimes Z^{s}$ have the property that $Z^{s}$ acting on $M_{\Gamma}$ extends to an action of $\boldsymbol{Z}^{s} \otimes \boldsymbol{R}$ acting on $M_{\Gamma}$ and preserving eigenvalue one subspaces. Then the group $R$ constructed as the solution to Problem 2 above has the property that $R \supset \Gamma$ and $R / \Gamma$ is compact. Thus we have proven the following theorem.

A necessary and sufficient condition that group $\Gamma$ satisfying (1) be a discrete cocompact subgroup of a connected simply connected solvable Lie group is that if $M_{\Gamma}$ is the algebraic hull of the nil-shadow of $\Gamma$ and $Z^{s}$ is the semisimple part of $\Gamma$ then the action of $Z^{s}$ in $M_{\Gamma}$ can be extended to $\boldsymbol{Z}^{s} \otimes \boldsymbol{R}$ and preserves the eigenvalue one subspaces.

This shows the central role played by the automorphism extension problem for compact solvmanifolds.

We are now in a position to combine the free nilpotent Lie groups with the above discussion to characterize the fundamental groups of compact solvmanifolds.

Let $\Gamma$ as usual satisfy (1) and let $\left(\Gamma_{R}\right)_{s}^{\tilde{s}}=H_{\Gamma} \rtimes Z^{s}$ and let $M_{\Gamma}$ be the algebraic hull of $H_{\Gamma}$. Viewing $M_{\Gamma}$ as a vector space, assume it is a subspace of a vector space $W$ such that $\boldsymbol{Z}^{s}$ extends semisimply to $W$ with the property that this action of $\boldsymbol{Z}^{s}$ in $W$ can be extended to $\boldsymbol{Z}^{s} \otimes \boldsymbol{R}$. (Note we can always take $W=M_{\Gamma} \otimes C$.) Let $M_{\Gamma}$ be $k$-step nilpotent and form $F^{k}(W)$. Choose a linear map $W \rightarrow M_{\Gamma}$ whose kernel $K$ is $\left(Z^{s} \otimes 1\right)$-invariant. Then we may view $Z^{s} \otimes R$ acting on $F^{k}(W)$ and so we may form $Z^{s} \otimes R \ltimes F^{k}(W)$ 
and let $\mathscr{I}(K)$ be the ideal in $F^{k}(W)$ generated by $K$. Then let $\mathscr{K}: F^{k}(W)$ $\rightarrow M_{\Gamma}$ be the homomorphism with kernel $\mathscr{I}(K)$. One checks that $\mathscr{K}$ extends to a homomorphism $\mathscr{K}^{*}: Z^{s} \otimes 1 \ltimes F^{k}(W) \rightarrow Z^{s} \ltimes M_{\Gamma}$. Now $\Gamma \subset Z^{s} \ltimes M_{\Gamma}$ and so we may consider $\mathscr{K}^{*-1}(\Gamma) \subset Z^{s} \otimes R \ltimes F^{k}(W)$. By the solution of Problem 2 there exists $\mathscr{R} \supset \mathscr{K}^{*-1}(\Gamma)$ and such that $\mathscr{R} \subset \boldsymbol{Z}^{s} \otimes \boldsymbol{R} \ltimes F^{k}(W)$ with the full group as its semisimple splitting. Clearly $\mathscr{R} / \mathscr{K}^{*-1}(\Gamma)$ is compact and $\mathscr{K}^{*-1}(\Gamma) / \mathscr{K}^{*-1}(\Gamma)_{0} \approx \Gamma$ and this proves our assertion that any group $\Gamma$ satisfying (1) is the fundamental group of a compact solvmanifold.

6. Presentations of compact solvmanifolds. Let $R$ be a connected, simply connected solvable Lie group and let $D$ be a closed subgroup of $R$ such that $R / D$ is compact. If $D$ has no connected subgroup normal in $R$, we will say that $R / D$ is a presentation of $X=R / D, X$ a compact solvmanifold. It is clear that the concept of presentation has been introduced to avoid an annoying but trivial complication. For if $H \subset D$ is connected and normal in $R$ then

$$
(R / H) /(D / H) \approx X
$$

and so we may replace $R$ by $R / H$ when writing the homogeneous space $R / D$.

With this definition out of the way, we may state the two problems that we will study in this section.

Problem 1. Find a necessary and sufficient condition for a solvable Lie group to have a discrete subgroup $\Gamma$ such that $R / \Gamma$ is compact.

Problem 2. Given a compact solvmanifold $X$ find all pairs $\left(R_{\alpha}, D_{\alpha}\right)$ such that $R_{\alpha} / D_{\alpha}$ is a presentation for $X$.

Both of these problems have been studied in some detail. The first by G. D. Mostow in $[\mathrm{M}, \mathrm{G} ; \mathbf{3}]$ and the second by R. Johnson in $[\mathrm{J}, \mathrm{R}]$. However our treatment of both problems is different than the above treatments. Indeed, to Problem 2 we will present a complete solution, up to isomorphism, which except for the torus Johnson failed to achieve. However he did show that there exist presentations $\left(S_{\alpha}, C_{\alpha}\right)$ such that $\operatorname{dim} S_{\alpha} \rightarrow \infty$ as $\alpha \rightarrow \infty$.

Let $R$ be a solvable Lie group with semisimple splitting $R_{s}^{\sim}=M_{R} \rtimes \boldsymbol{R}_{R}$. Necessary and sufficient conditions for $R$ to contain a discrete cocompact subgroup are the following:

(1) $M_{s}$ has a rational form $M_{Q}$ such that if $N$ is the nil-radical $N$ of $R$ then $M_{\mathbf{Q}} \cap N$ is a rational form of $N$.

(2) Consider $M_{R} / N \oplus R^{s}$ which contains $R / N$ as a subgroup. Let $p_{1}: M_{\boldsymbol{R}} / N \oplus \boldsymbol{R}^{s} \rightarrow M_{\boldsymbol{R}} / N$ and $p_{2}: M_{\boldsymbol{R}} / N \oplus \boldsymbol{R}^{s} \rightarrow \boldsymbol{R}^{s}$. Then $p_{1} \mid \boldsymbol{R} / N=q_{1}$ and $p_{2} \mid R / N=q_{2}$ are linear isometries. Let $q_{2} q_{1}^{-1}\left(M_{\boldsymbol{Q}} / N\right)$ be $\Phi \subset \boldsymbol{R}_{s}$. Then $\Phi$ contains a lattice subgroup $L$ such that $\boldsymbol{R}_{s} / L$ is compact and $L$ acts as integer 
unimodular matrices on a basis of $M_{\mathbf{Q}}$.

The necessity follows easily by replacing a discrete subgroup by a lattice nilpotent subgroup. The sufficiency follows from the fact proven about lattice nilpotent subgroups invariant under groups of integer unimodular matrices of Chapter II, $\S 3$ and the diagonal arguments of the previous section.

Our solution to Problem 2 is also very simple once we are in possession of the Mostow structure theorem for presentations of compact solvmanifolds. We will state it and use it now although we will delay its proof to Chapter IV, §3. The structure theorem says the following: Let $R$ be a solvable Lie group and let $D$ be a closed subgroup such that $R / D$ is a presentation. Let $N$ be the nil-radical of $R$. Then $D$ satisfies

$$
1 \rightarrow N \cap D \rightarrow D \rightarrow Z^{s} \rightarrow 1
$$

where $N$ is the algebraic hull of $N \cap D$ and $Z^{s}$ is a lattice in $R / N$.

If we form $\left(D_{R}\right)_{s}^{\tilde{s}}=H_{D} \ltimes Z^{s}$ and $M_{D}$ is the algebraic hull of $H_{D}$ then $R_{s}^{\sim}=M_{D} \ltimes\left(Z^{s} \otimes R\right) \rho$ where as usual $\left(Z^{s} \otimes R\right) \rho$ denotes an action of $Z^{s} \otimes R$ on $M_{D}$. Now let $D_{0}$ be the identity compact of $D$ then $D_{0}$ is a normal subgroup of $M_{D}$ and $H_{D}$ and if $\Gamma=D / D_{0}$, then

$$
\left(\Gamma_{R}\right)_{s}^{\tilde{s}}=H_{D} / D_{0} \rtimes Z^{s}
$$

and $M_{D} / D_{0}$ is the algebraic hull of $H_{D} / D_{0}$.

Now let $M_{D}$ be the $k$-step nilpotent and let $\sigma: F^{k}\left(M_{D}\right) \rightarrow M_{D}$ be the canonical mapping obtained from the identity mapping. Then since $\left(Z^{s} \otimes R\right) \rho$ operates on $M_{D}$ we may extend $\sigma$ to a homomorphism

$$
\sigma:\left(\boldsymbol{Z}^{s} \otimes R\right) \rho \ltimes F^{k}\left(M_{D}\right) \rightarrow\left(Z^{s} \otimes R\right) \rho \ltimes M_{D}
$$

and let $\sigma^{-1}(R)=\mathscr{R}$.

Now since $Z^{s} \otimes 1$ operates semisimply in $M_{D}$ leaving $D_{0}$ invariant we have

$$
\left(Z^{s} \otimes 1\right) \rho \ltimes F^{k}\left(M_{D}\right) \stackrel{\mu}{\rightarrow} M_{D} / D_{0} \rtimes Z^{s}
$$

is well defined. Now $\Gamma \subset M_{D} / D_{0} \rtimes Z^{s}$ and $\mu^{-1}(\Gamma)$ is cocompact in $\mathscr{R}$. It is straightforward to now verify that $R$ is obtained from $\mathscr{R}$ by dividing out by the maximal connected subgroup of $\mu^{-1}(\Gamma)$ that is normal in $\mathscr{R}$. This shows that the construction given in the previous section can be made into a generic construction for all presentations of a compact solvmanifold by dividing $\mathscr{R}$ by the maximal connected subgroup of $\mathscr{K}^{*-1}(\Gamma)$ that is normal in $\mathscr{R}$.

Thus we see that the presentation problem for a compact solvmanifold reduces to an extension problem and an isomorphism problem.

7. Compact solvmanifolds with discrete isotropy groups. Let $\Gamma$ be a 
discrete subgroup of a connected nilpotent group $N$ such that $N / \Gamma$ is compact. Then, by the results of Chapter II, we saw that $N$ is unique. But, in Example 4 of Chapter III, $\S 1$, we saw that if $\widetilde{R}$ denotes the universal covering group of the group of rigid motions of the plane, then $\widetilde{R}$ operates transitively and with discrete isotropy group on the three-dimensional torus. Hence a torus may be written in more than one way in the form $R / \Gamma$ where $R$ is a solvable Lie group and $\Gamma$ is a discrete subgroup. The basic question of this section is given two presentations $R_{1} / \Gamma$ and $R_{2} / \Gamma$ of a compact solvmanifold with discrete isotropy group $\Gamma$, how much structure do $R_{1}$ and $R_{2}$ have in common? (It will be clear from the discrete case what will be true for the closed subgroup case so we will not present the details.)

This problem has been studied in $[A, L ; 3,12,10]$ and $[T, R ; 2]$. We will use the semisimple splitting, in the spirit of $[T, R ; 2]$, to unify these ideas and present a true uniqueness theorem that has already been used to study the automorphism groups of polycyclic groups $[\mathrm{A}, \mathrm{L} ; \mathbf{1 4}]$.

Let $X=R_{i} / \Gamma, i=1,2$, and let us write $X$ in the affine presentation $X=M / a(\Gamma)$, where $M$ is the algebraic hull of the nil-shadow of the Wang splitting of $\Gamma_{R}$. Further $a(\Gamma)$ is a faithful representation of $\Gamma$ in $A(M) \ltimes M$. Now we know from Chapter III, $\S 4$ that $\left(R_{i}\right)_{s}=T_{i} \ltimes M, i=1$, 2, where $T_{i} \subset A(M)$ is completely reducible and abelian.

Now let $\alpha: A(M) \ltimes M \rightarrow A(M)$ be the homomorphism with kernel $M$ and let $A^{0}$ denote the identity component of the algebraic hull of $\alpha \circ a(\Gamma)$. If $\Gamma$ satisfies

$$
1 \rightarrow \pi \rightarrow \Gamma \rightarrow Z^{s} \rightarrow 1
$$

where $\pi$ is nilpotent then $\pi_{R} \subset M$. Further $A^{0}$ acts trivially on $M / \pi_{R}$ since $\Gamma$ does. Now $\alpha\left(T_{i}\right)$ acts trivially on $q(\Gamma)$ and so $\alpha\left(T_{i}\right)$ acts trivially on $A^{0}$. It follows easily from this that $\alpha\left(T_{i}\right) A^{0}=C_{i} \oplus A^{0}$, where $C_{i}$ is a compact abelian subgroup of $A(M)$.

Now let $\Gamma_{0} \subset \Gamma$ be the subgroup of $\Gamma$ which is the pre-image of the group $\alpha(\Gamma) \cap A^{0}$. In [A, L; 10, 14], I defined a subgroup of finite index of $\Gamma_{0}$ and called such groups "algebraic strongly torsion free $S$ groups of type $T$ " and "predivisible" polycyclic groups in $[\mathrm{A}, \mathrm{L} ; 10]$ and $[\mathrm{A}, \mathrm{L}$; 14] respectively. For the rest of this paper we will call $\Gamma_{0}$ "the predivisible subgroup of $\Gamma$ " and if $\Gamma$ is its own predivisible subgroup we will call $\Gamma$ a predivisible polycyclic group.

Let $\Gamma$ be a predivisible polycyclic group.

Since $A^{0}$ is an abelian connected Lie group, $A^{0}$ has a unique maximal compact subgroup $C$ and so we may form $A^{\circ} / C$ and consider the image $\alpha \circ a(\Gamma)$ in $A^{0} / C$. Let $W$ be the linear hull of the image of $\alpha \circ a(\Gamma)$ in $A^{0} / C$ and let $B$ be the pre-image of $W$ in $A^{0}$. Let $D^{\#}(\Gamma)=M_{\Gamma} \rtimes B$. Now let $\pi \supset[\Gamma, \Gamma]$ and be such that $\pi$ is nilpotent and satisfies the exact sequence 


$$
1 \rightarrow \pi \rightarrow \Gamma \rightarrow Z^{s} \rightarrow 1 .
$$

Let $\pi_{\boldsymbol{R}}$ be the algebraic hull of $\pi$. Then $\pi_{\boldsymbol{R}}$ is normal in $D^{\#}(\Gamma)$ and $D^{\#}(\Gamma) / \pi_{\boldsymbol{R}}$ is abelian. Thus we have a well-defined isomorphism

$$
\gamma: \Gamma / \pi \rightarrow M_{\Gamma} \rtimes B / \pi_{R}
$$

which, since the right side is a connected abelian group, can be extended to a continuous isomorphism

$$
\gamma^{*}: \Gamma / \pi \otimes R \rightarrow M_{\Gamma} \rtimes B / \pi_{\boldsymbol{R}}
$$

Although $\gamma^{*}(\Gamma / \pi \otimes R)=D^{*}$ is not unique, the group $D^{*} C$ contains $\gamma^{*}(\Gamma / \pi \otimes R)$ for all possible choices of extensions. Let $\eta: M_{\Gamma} \rtimes B \rightarrow M_{\Gamma}$ $\rtimes B / \pi_{R}$ and let $\eta^{-1}\left(D^{*}\right)=R(\Gamma)$ and $\eta^{*}\left(D^{*} C\right)=D(\Gamma)$. Clearly

1. $D(\Gamma) \supset \Gamma$ as discrete cocompact subgroup and $D(\Gamma) / \Gamma$ is compact. Although $D(\Gamma)$ is a solvable Lie group, it is not simply connected in general.

2. $R(\Gamma) \supset \Gamma$ and $R(\Gamma) / \Gamma$ is compact. Although $R(\Gamma)$ is simply connected, it is not unique.

3. $D(\Gamma)=C \ltimes R(\Gamma)$ where $C$ is a compact abelian group.

The groups $D(\Gamma)$ were introduced and studied in $[\mathrm{A}, \mathrm{L} ; 14]$. From the point of view adopted here Theorems 1 and 2 of that paper become trivial consequences of the definitions and may be stated as follows: Let $\Gamma$ be a predivisible group and let $D(\Gamma)$ be defined as above and called the splitLie hull of $\Gamma$.

1. Every automorphism of $\Gamma$ uniquely extends to an automorphism of $D(\Gamma)$.

2. Let $G$ be a group that contains $\Gamma$ as a normal subgroup of finite index and let $F=G / \Gamma$. Then if the group $G D(\Gamma)$ is defined by the commutative diagram

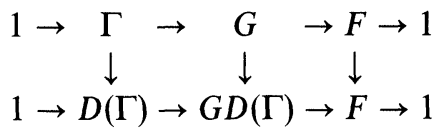

the bottom exact sequence splits.

The groups $R(\Gamma)$ were first introduced in $[\mathrm{A}, \mathrm{L} ; \mathbf{1 0}]$ where their existence and basic properties were first established. Again using the point of view adopted here Theorems 1 and 2 of $[\mathrm{A}, \mathrm{L} ; \mathbf{1 0}]$ become trivial consequences of the definition.

1. $R(\Gamma)$ contains $\Gamma$ as a discrete cocompact subgroup.

2. Let $\theta$ be an automorphism of $\Gamma_{\mathbf{R}}$. Then if $\theta$ is trivial in $\Gamma_{\boldsymbol{R}} / \pi_{\boldsymbol{R}}, \theta$ is uniquely extendable to an automorphism of $R(\Gamma)$.

Until now we have not stated results using exact sequences. Such results are of course implicit in what we have already proven and we will 
now make them explicit. The result below generalizes the results in $[\mathrm{A}, \mathrm{L}$; 3, 16].

Let $R_{1}$ and $R_{2}$ be connected, simply connected solvable Lie groups with nil-radicals $N_{1}$ and $N_{2}$ respectively. Further, let $\Gamma_{i}$ be discrete cocompact subgroups of $R_{i}, i=1,2$, and let $\alpha$ be an isomorphism of $\Gamma_{1}$ and $\Gamma_{2}$ with the following properties:

1. $\alpha$ induces an isomorphism $\alpha_{1}$ of $\Gamma_{1} \cap N_{1}$ onto $\Gamma_{2} \cap N_{2}$ and so $\alpha$ uniquely extends to an isomorphism $\alpha_{1}^{*}$ of $N_{1}$ onto $N_{2}$.

2. Let $V_{i}=R_{i} / N_{i}, i=1,2$, and let $\alpha$ induce the isomorphism $\alpha_{2}$ of $\Gamma_{1} / \Gamma_{1} \cap N_{1}$ and $\Gamma_{2} / \Gamma_{2} \cap N_{2}$. Then $\alpha_{2}$ can be extended uniquely to give an isomorphism $\alpha_{2}^{*}$ of $V_{1}$ onto $V_{2}$. Further, we assume that

$$
\begin{array}{cc}
V_{1} \rightarrow A\left(N_{1}\right) / \mathscr{I}\left(N_{1}\right) \\
\alpha_{2}^{*} \downarrow & \downarrow \alpha_{1}^{* *} \\
V_{2} \rightarrow A\left(N_{2}\right) / \mathscr{I}\left(N_{2}\right)
\end{array}
$$

where the horizontal arrows are induced by coset actions is commutative where $\mathscr{I}(\quad)$ is the group of inner automorphisms of the group in the bracket and $\alpha_{1}^{* *}$ is determined from $\alpha_{1}^{*}$.

Under all the above conditions $R_{1}$ is isomorphic to $R_{2}$.

We may see the assertion is true by noting that $\alpha$ induces an isomorphism of $\left(R_{1}\right)_{s}$ onto $\left(R_{2}\right)_{s}$. The rest is then trivial.

\section{CHAPTER IV : DISCRETE SEMISIMPLE SPLITTING AND APPLICATIONS}

1. Introduction. Let me begin by explaining the reason for the length and detail of the next section. When I first proved the Philip Hall conjecture $[\mathrm{A}, \mathrm{L} ; \mathrm{15}], \mathrm{H}$. C. Wang found a way of shortening the proof. The shortened version was the one that was published in $[A, L ; 7]$. The published version does not show how the Hall conjecture can be derived from a discrete semisimple splitting theorem. Instead, one directly proves the Hall conjecture. Later it was stated that one can derive the discrete semisimple splitting theorem from the Hall conjecture. Since it is my belief that the discrete semisimple splitting is the key tool, I will reproduce here a refined version of my original proof. This starts by directly proving the existence of a discrete splitting theorem and deduces from it the Hall conjecture. We also use the discrete splitting theorem to prove Mostow's structure theorem for presentations of compact solvmanifolds.

Before plunging ahead with the details of this discussion let us pause to resumé some facts and notations that have already been established.

Let $N$ be a connected, simply connected nilpotent Lie group. A closed subgroup of $N$ will be called a $C N$ group. We know from Chapter II that a $C N$ group $\Delta$ can be characterized as a torsion free nilpotent topological group whose identity component $\Delta_{0}$ is a connected, simply connected Lie group and such that $\Delta / \Delta_{0}$ is finitely generated. Further, if $\Delta$ is a $\mathrm{CN}$ 
group, there exists a unique nilpotent Lie group $\Delta_{R}\left(\Delta_{Q}\right)$ such that $\Delta_{R} \supset \Delta$ and $\Delta_{\boldsymbol{R}} / \Delta$ is compact $\left(\Delta_{\boldsymbol{Q}} / \Delta_{0}\right.$ is a rational nilpotent Lie group and the real completion of $\Delta_{\boldsymbol{Q}}$ is $\Delta_{R}$ ). If $\Delta$ is a $\mathrm{CN}$ group with trivial identity component we will call $\Delta$ an FN group.

We have seen in Chapter III that $\Gamma$ is the fundamental group of a compact solvmanifold if and only if $\Gamma$ satisfies an exact sequence

$$
1 \rightarrow \Delta \rightarrow \Gamma \rightarrow Z^{s} \rightarrow 1
$$

where $\Delta$ is an FN group. Fundamental groups of compact solvmanifolds will be called FS groups. If $\Delta$ in (1) is a $\mathrm{CN}$ group we will call $\Gamma$ a CS group. If $\Gamma$ is a CS group satisfying (1), there exists a unique group $\Gamma_{\mathbf{Q}}\left(\Gamma_{R}\right)$ satisfying the commutative diagram

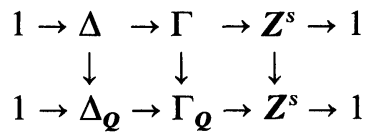

( $\Gamma_{R}$ is defined as above with $\Delta_{R}$ replacing $\Delta_{\mathcal{Q}}$ ).

2. Discrete semisimple splittings. Let us begin by formally stating the main existence theorem. that

Let $\Gamma$ be a CS group. Then there exists a closed subgroup $\Delta^{*}$ of $\Delta_{\boldsymbol{Q}}$ such

(a) $\Delta^{*} \supset \Delta$ and $\Delta^{*} / \Delta$ is finite,

(b) $\Delta^{*}$ is normalized by $\Gamma$.

Further if we let $\Gamma^{*}=\Gamma \Delta^{*}$ and let $\left(\Gamma_{R}\right)_{s}=T_{\Gamma} \ltimes H_{\Gamma}$, then we may choose $n \in H_{\Gamma}$ such that $n T_{\Gamma} n^{-1}=T$ has the property that

(c) $\Gamma^{*}$ and $\Delta^{*}$ are invariant under $T$.

Given a CS group $\Gamma$ the group $T \times \Gamma^{*}$ whose existence we have just asserted is called a discrete semisimple splitting of $\Gamma$.

We will only prove the existence of a discrete semisimple splitting of $\Gamma$ under the restriction that $\Gamma$ has trivial identity component. The proof in the general case goes through in essentially the same way or may be reduced to the special case treated here.

Let us begin by remarking on the following: Let $\Gamma$ satisfy

$$
1 \rightarrow \Delta \rightarrow \Gamma \rightarrow Z^{s} \rightarrow 1
$$

where $\Delta$ is an FN group. Let $\Delta_{*}$ be the lattice hull of $\Delta$. Then there exists a unique group $\Gamma_{*}$ satisfying the following commutative diagram:

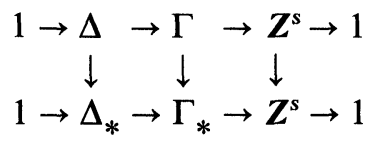

Further any automorphism of $\Gamma$ is uniquely extendable to $\Gamma_{*}$. We will call $\Gamma_{*}$ a lattice polycyclic group. 
We next need the following fairly straight forward result. Let

$$
1 \rightarrow \Delta \rightarrow \Gamma \rightarrow Z^{s} \rightarrow 1
$$

be a lattice polycyclic group and let $\left(\Gamma_{R}\right)_{s}^{\sim}=H_{\Gamma} \rtimes Z^{s}$. Let $e_{1}, \ldots, e_{r}$ be a basis of $L\left(\Delta_{R}\right)$ that generates $\log (\Delta)$ as a $Z$ module. Then using the cross section $f$ and the mapping $g$ introduced in Chapter III, $\$ 4$, we have $\operatorname{ad}(\mathrm{g}(y))$, $y \in Z^{s}$, is rational relative to $e_{1}, \ldots, e_{r}$ and $\left[g\left(y_{1}\right), g\left(y_{2}\right)\right] \in \Delta$ for $y_{1}, y_{2} \in Z^{s}$.

This assertion may be verified as follows. Since $\log (\Delta)$ is a lattice in $L\left(\Delta_{R}\right)$, and $\operatorname{ad}(f(g))$ preserves $\log (\Delta), g \in Z^{s}$, we have $\operatorname{ad}(f(g))$ is integer unimodular and so its semisimple and unipotent parts are rational. But $\operatorname{ad}(g(y)), y \in Z^{s}$, is exactly the unipotent part of $\operatorname{ad}(f(y))$.

To see that $\left[g\left(y_{1}\right), g\left(y_{2}\right)\right] \in \Delta$ we just have to see that $\left[f\left(y_{1}\right), f\left(y_{2}\right)\right] \in \Delta$ and that $\operatorname{ad}\left(f\left(y_{1}\right)\right)=b_{i} \operatorname{ad}\left(g\left(y_{i}\right)\right), b_{i}$ semisimple and acting trivially on $g\left(y_{i}\right)$ all $y_{i} \in Z^{s}$.

Let us now state the discrete splitting theorem we will actually prove and give a proof for it.

Let $\Gamma$ be a lattice polycyclic group and let $\left(\Gamma_{R}\right)_{s}^{\sim}=H_{\Gamma} \times Z^{s}$. Then $H_{\Gamma}$ contains a discrete cocompact subgroup $D$ such that

(a) $D$ is a lattice nilpotent group,

(b) $D \cap \Delta_{R} \supset \Delta$,

(c) $D$ contains the set $g\left(Z^{s}\right)$ in the above notation,

(d) $Z^{s}$ acting on $H_{\Gamma}$ maps $D$ onto itself.

The existence of $D$ satisfying (a), (b), and (c) follows easily from the fact that $\left(H_{\Gamma}\right)_{R}$ is rational nilpotent relative to the appropriate basis of $L\left(\left(H_{\Gamma}\right)_{R}\right)$. It is thus condition (d) that is the essential condition. We will prove this by a slight modification of the argument given in Chapter II, §3. Let $M=\left(H_{\Gamma}\right)_{R}$ and let $M_{k}$ be the last nontrivial subgroup in the lower central series of $M$. Then $H_{\Gamma} / M_{k} \cap H_{\Gamma} \rtimes Z^{s}$ is the Wang splitting of $\Gamma / M_{k \cap \Gamma}$. Hence by induction we may find $D^{\prime}$ in $M / M_{k}$ satisfying the conditions of our theorem. Now $Z^{s}$ acting on $M_{k}$ is integer unimodular relative to $\Delta \cap M_{k}$. But then the construction of lattice nilpotent subgroups discussed in Chapter II, $\S 3$ assures us that the group generated by a pre-image of $D^{\prime}$ in $M$ and $\left(D \cap M_{k}\right) / e$, some $e \in Z^{+}$, will be a lattice nilpotent subgroup of $M$ invariant under $Z^{s}$. This proves our assertion.

Given a group $\Gamma$ the group $Z^{s} \ltimes D$ constructed above will be called a discrete splitting of $\Gamma$. It is easy to see that the intersection of all $D$ 's satisfying the conclusions of the above theorem also satisfies the theorem. Henceforth we will restrict ourselves to this minimal $D$ and call $Z^{s} \ltimes D$ the discrete semisimple splitting of $\Gamma$. It is easily verified that every automorphism of $\Gamma$ extends uniquely to its discrete semisimple splitting.

3. The P. Hall conjecture and Mostow's structure theorem for solvmanifolds. In this section we will prove the P. Hall conjecture and then 
the Mostow structure theorem for presentations of solvmanifolds as corollaries of the discrete semisimple splitting theorem. In Chapter III, $\$ 3$ we discussed the Birkhoff imbedding theorem $[B, G]$. We will need the following slight refinement of the results stated in Chapter III, \$3. Let $L$ be a nilpotent Lie algebra and let $e_{1}, \ldots, e_{r}$ be a basis of $L$ with integer constants of structure. Then the Birkhoff imbedding of $L, B(L)$, will have the property that $B\left(e_{i}\right), i=1, \ldots, r$, are integer nilpotent matrices. Further if $A$ is an automorphism of $L$ which is integer unimodular relative to $e_{1}, \ldots, e_{r}$ then $B(A)$ is an integer unimodular matrix.

The following is the key step in proving the P. Hall conjecture from one point of view.

Let $D$ be a lattice nilpotent polycyclic group and let $K$ be a group of automorphisms of $D$. Then there exists a subgroup $D^{\#}$ of finite index in $D$ such that $D$ is invariant under $K$ and $K \ltimes D^{\#}$ has a faithful representation in $G L(n, Z)$.

Let $e_{1}, \ldots, e_{r}$ be a basis of $L\left(D_{R}\right)$ be such that the $Z$-module generated by this basis is $\log (D)$. Since the constants of structure of $L\left(D_{R}\right)$ are rational relative to $e_{1}, \ldots, e_{r}$, there exists an integer $d_{1}$ such that $L\left(D_{R}\right)$ has integer constants of structure relative to $d_{1} e_{1}, \ldots, d_{1} e_{r}$. Further, $K$ acting on $L\left(D_{R}\right)$ is still integer unimodular relative to the basis $d_{1} e_{1}, \ldots, d_{1} e_{r}$. Hence the Birkhoff imbedding $B$ of $L\left(D_{R}\right)$ maps $d_{1} \log (D)$ into nilpotent integer matrices and $B(K)$ is a group of integer unimodular matrices. Let Exp denote matrix exponentiation. Then it is easy to see that $\operatorname{Exp}\left(d_{1} \log (D)\right) \cap G L(n, Z)=D^{+}$is commensurable with $D$ and $K$ invariant. Thus clearly $D \cap G L(n, Z)=D^{\#}$ has the same property.

$P$. Hall conjectured that every polycyclic group $G$ has a faithful representation in $G L(n, Z)$. We are now in a position to prove this conjecture.

We first note that, using induced representations, it is enough to prove that a group commensurable with $G$ satisfies the Hall conjecture to conclude the Hall conjecture is true in general. It is a fact, essentially proven by Malcev [M, A; 3], that any polycyclic group is commensurable with a lattice polycyclic group $\Gamma$. Imbed $\Gamma$ in its discrete semisimple splitting $\boldsymbol{Z}^{s} \ltimes D$. Then by the above $\boldsymbol{Z}^{s} \ltimes D$ has a commensurable subgroup imbeddable in $G L(n, Z)$. This proves that $\Gamma$ has a commensurable subgroup imbeddable in $G L(n, Z)$ and thus we have proven the P. Hall conjecture.

We now turn to the proofs of two theorems of G. D. Mostow. The first theorem may be stated as follows:

Let $R$ be a connected, simply connected solvable Lie group and let $H$ be a closed subgroup of $R$ such that $R / H$ is a presentation of a compact solvmanifold. If $N$ is the nil-radical of $R$ then $H N / N$ is a discrete subgroup of $R / N$. 
Let us first reformulate this assertion using the language of semisimple splittings. Since $R / H$ is compact, we have by the Mostow conjecture that the real points of the nil-shadow of the semisimple splitting of $H_{R}$ is the nil-shadow of $R$. Let us introduce the notation $(R)_{s}=M_{R} \rtimes T_{R}$ and $H_{R}=M_{H} \rtimes T_{H}$ with $M_{H} \subset M_{R}$ and $T_{H} \subset T_{R}$. It is now trivial to verify that the following holds: $H N / N$ is a discrete subgroup of $R / N$ if and only if $T_{H}$ is a discrete subgroup of the group $A\left(M_{R}\right)$. Now let $M_{0}$ denote the nil-shadow of $H_{0}$. Let us now check that if $R / H$ is a presentation of a compact solvmanifold, then the natural map $q: T_{H} \rightarrow A\left(M_{R} / M_{0}\right)$ has trivial kernel.

To see this, let $t \in T_{H}$ be in the kernel of $q$. Then the range $X$ of $(t-I)$, where $I$ is the identity on $M_{R}$, is such that $X \cap M_{0}=X^{\prime}$ is nontrivial. Since $T_{R}$ is abelian $X$ and $M_{0}$ are invariant subspaces of $M_{R}$ under $T_{R}$. Hence the ideal $I\left(X^{\prime}\right)$ generated by $X^{\prime}$ in $M_{R}$ is in $M_{0}$ and invariant under $T_{R}$. This means that $I\left(X^{\prime}\right)$ is an ideal in $R_{s}$ and certainly in $R$. Since $R / H$ is a presentation, this implies that $X^{\prime}=0$ and the kernel of $q$ is trivial.

Now we can prove the Mostow structure theorem. The proof is that since $q\left(T_{H}\right)$ preserves a discrete cocompact subgroup of $M_{R} / M, q\left(T_{H}\right)$ is a discrete subgroup of $A\left(M_{R} / M_{0}\right)$ and hence certainly of $A\left(M_{R}\right)$.

Our final assertion of this section may be stated as follows: Let $R$ be a connected, simply connected solvable Lie group with discrete cocompact subgroup $\Gamma$. Then there exists a faithful representation $\zeta$ of $G$ in $G L(n, R)$ such that $\zeta(G) \cap G L(n, Z)$ is commensurable with $\Gamma$. To see this let $R_{s}=M_{R} \rtimes T_{R}$ be the semisimple splitting of $R$ and let $\Gamma_{s}=\Delta_{\Gamma} \rtimes T_{\Gamma}$ be the discrete semisimple splitting of $\Gamma$ with $\Delta_{\Gamma} \subset M_{R}, T_{\Gamma} \subset T_{R}$. Now $\left(\Delta_{\Gamma}\right)_{R}=M_{R}$. Hence the Birkhoff imbedding imbeds $R_{s}$ and $\Gamma_{s}$ in $G L(n, R)$ with $\Gamma_{s} \cap G L(n, Z)$ commensurable with $\Gamma_{s}$. This proves our assertion.

4. Solvable Lie groups of exponential type. There are two main classes of solvable Lie groups that have been studied. These are the exponential groups (groups of type $E$ ) and the type $R$ solvable groups (groups of type $R$ ). We will discuss groups of type $E$ in this section and type $R$ groups in the next section.

A connected, simply connected solvable Lie group $R$ is said to be of exponential type if the exponential map of $L(R)$ to $R$ is a homeomorphism. The simplest solvable Lie group that is not of type $E$ is the universal covering group of the group of rigid motions of the euclidean plane, $\widetilde{R}$. This may be seen by noting that $\widetilde{\mathscr{R}}$ has a matrix representation

$$
\left(\begin{array}{cccc}
\cos 2 \pi t & \sin 2 \pi t & 0 & x \\
-\sin 2 \pi t & \cos 2 \pi t & 0 & y \\
0 & 0 & 1 & t \\
0 & 0 & 0 & 1
\end{array}\right),
$$


$x, y, t \in \boldsymbol{R}$. It is easy to check that the element $x=\frac{1}{2}, y=0, t=1$ has no square root in $\widetilde{R}$.

Groups of type $E$ have been characterized of J. Dixmier $[\mathrm{D}, \mathrm{J}]$ and $\mathrm{M}$. Saito [S, M]. Clearly if $R$ is type $E$ both any connected subgroup of $R$ is type $E$ and any simply connected homomorphic image of $R$ is type $E$. (Note the first assertion follows from the semisimple splitting of $R$ and the fact that nilpotent groups obviously have this property.) Hence it is clear that $R$ is not of type $E$ if it has a connected subgroup $H$ such that $H$ has a homomorphism onto $\widetilde{R}$. The next theorems show that this condition is also necessary and give a simple criterion for $R$ not to be of type $E$.

Let $R$ be a connected, simply connected solvable Lie group with semisimple splitting $R_{s}=M_{R} \rtimes T_{R}$. Then there exists $t \in T_{R}$ which has an eigenvalue $\theta$ such that $\theta \neq 1$ but $|\theta|=1$ if and only if $R$ has a connected subgroup $H$ which has a homomorphism onto $\widetilde{R}$.

Let us begin by assuming that $H \subset R$ exists such that $\gamma: H \rightarrow \widetilde{R}$ is a surjection. Let $H_{s}=M_{H} \rtimes T_{H}$ be a semisimple splitting so chosen that $M_{H} \subset M_{R}$ and $T_{H} \subset T_{R}$. Let the kernel of $\gamma$ be $K$. Note that $K \cap M_{H}$ is normal in $H$ and so invariant under $T_{H}$. Hence the action of $T_{H}$ on the complementary invariant subspace in $M_{H}$ to $K \cap M_{H}$ is the same as the semisimple part of $\operatorname{ad}(\widetilde{\mathscr{R}})$. Thus $T_{H}$ has an element $t$ with an eigenvalue $\theta$ such that $|\theta|=1$ but $\theta \neq 1$.

Conversely, let $t \in T_{H}$ act on $M_{H}$ with an eigenvalue $\theta$ such that $|\theta|=1$ but $\theta \neq 1$. Let $\left(M_{H}\right)_{i}, i=1, \ldots, k$, be the lower central series of $M_{H}$. Then there is a largest index $j$ such that $t \mid\left(M_{H}\right)_{j}$ has eigenvalue $\theta$ with $|\theta|=1$ and $\theta \neq 1$. Then notice that $T_{H}$ is connected and hence there are $t_{0} \in T_{H}$ arbitrarily close to the identity with the same property for its eigenvalues. Hence there is in $R$ a one parameter group $p(s)$ such that the semisimple part of $p(s)$ contains $t_{0} \in T_{H}$. This is because the exponential map is a homeomorphism in some neighborhood of the identity.

Now form the subgroup $p(t) \ltimes\left(M_{H}\right)_{j} \subset R$. It is easily seen that $p(t) \ltimes\left(M_{H}\right)_{j}$ has a homomorphism onto $\mathscr{R}$. Indeed in $L\left(\left(M_{H}\right)_{j}\right)$ choose any subspace $W$ containing $L\left(\left(M_{H}\right)_{j+1}\right)$ and of codimension 2 in $L\left(\left(M_{H}\right)_{j}\right)$ with the property that it is $p(t)$ invariant and $\operatorname{ad}(p(t))$ has eigenvalue of absolute value 1 , but not constantly 1 , on the complement of $W$. Clearly, $\exp (W)$ is a normal subgroup of $p(t) \ltimes\left(M_{H}\right)_{j}$ with quotient group $\widetilde{\mathscr{R}}$.

We will now give a criterion that tells when a solvable Lie group is of type $E$.

Let $R$ be a connected, simply connected solvable Lie group with semisimple splitting $R_{s}=M_{R} \rtimes T_{R}$. Then $R$ is type $E$ if and only if $T_{R}$ has no eigenvalues such that $|\theta|=1$ but $\theta \neq 1$.

By our previous discussion if $T_{R}$ has an eigenvalue $\theta$ such that $|\theta|=1$ but $\theta \neq 1$, then $R$ has a subgroup $H$ with a surjection onto $\widetilde{R}$ and so $R$ 
is not type $E$. Hence it only remains to verify the converse.

Now let $T_{R}$ have no eigenvalue $\theta$ such that $|\theta|=1$ but $\theta \neq 1$. Then it is easily verified that $T_{R}$ is simply connected and if $t_{0} \in T_{R}$ with eigenvector $v$ with eigenvalue 1 and $t(s) \subset T_{R}$ is the one parameter group through $t_{0}$ then $t(s)(v)=v$ for all $s$. Now let $r \in R$ and let $R_{s}=M_{R} \rtimes T_{R}$ be chosen so that $r=m t$ with $m \in M_{R}, t \in T_{R}$ and $m t=t m$. Let $t(s)$ be the one parameter group in $T_{R}$ such that $t(1)=t$ and $m(s)$ the one parameter group in $M_{R}$ such that $m(1)=m$. Then it is easily verified that $m(s) t(s)=t(s) m(s)$ and so is a one parameter group and also that $m(s) t(s) \subset R$.

In the course of the above discussion we showed that if $R$ is of type $E, T_{R}$ is simply connected. The converse to this assertion is easily seen to be false.

Our discussion above has four important but easily verified consequences (in the order presented) by us. Let $R$ be a connected, simply connected group of exponential type and let $\Gamma$ be a discrete subgroup of $R$.

(1) Let $h(\Gamma)$ be the intersection of all the connected subgroups of $R$ which contain $\Gamma$. Then $R / \Gamma$ is compact if and only if $h(\Gamma)=R$.

(2) Let $r \in R$, let $p(s)$ be the one parameter group such that $p(1)=r$ and let $X$ be the tangent space to $p(s)$ at $s=0$. Then $[X, L(R)]=$ $L([p(s), R])=\operatorname{range}(\operatorname{ad}(r)-I) L(R)$.

(3) Let $G$ be a connected subgroup of $R$ such that $G / \Gamma \cap G$ is compact. Then $[L(G), L(R)]=L[G, R]=\operatorname{span} \bigcup\left\{\left(\operatorname{ad}\left(\gamma_{i}\right)-I\right) L(R)\right\}$ where $\gamma_{i} \in \Gamma \cap G$ and the one parameter groups through $\gamma_{i}$ have tangent vectors that span $L(G)$.

(4) Let $V$ be a connected abelian subgroup of $R$ such that $\Gamma \cap V=L$ is cocompact in $V$. Then $\Gamma \cap[R, V]$ is compact in $[R, V]$. (Note in proving this last assertion that, for $\gamma \in \Gamma,(\operatorname{ad}(\gamma)-I) L$ is a discrete cocompact subgroup of $(\operatorname{ad}(\gamma)-I) V$. $)$

We will now discuss the result we have been aiming towards in this discussion. This result was first presented in [A, L; 17, Appendix to VII]. On rereading the proof it seems confusing to me and not all assertions are correct. However the argument in broad outline seems to me to be about the same as presented here.

Let $R$ be an exponential solvable Lie group and let $\Gamma$ be a discrete cocompact subgroup. Let $G$ be a connected normal subgroup of $R$ such that $\Gamma \cap G$ is cocompact in $G$. Then $[R, G] / \Gamma \cap[R, G]$ is compact.

Let $[G, G]=N$ and let $M$ be the nil-radical of $G$. Then $\Gamma \cap M$ is cocompact in $M$ and hence $\Gamma \cap[M, M]$ is cocompact in $[M, M]$. Since $[M, M]$ is characteristic in $M$ it is normal in $R$ and so, by induction, it is easily seen we may reduce to the case where $[M, M]=e$.

We may now use assertion 4 above to conclude that $\Gamma \cap[G, G]$ is cocompact in $[G, G]$ and so we may again assume that $[G, G]=e$. But 
then $G$ is abelian and applying 4 again completes the argument.

If we define

$$
\left.R^{\infty}=\lim _{k \rightarrow \infty}[\underbrace{R \cdots[R}_{k}, R] \cdots\right]
$$

we have by simple induction that $\Gamma \cap R^{\infty}$ is cocompact in $R^{\infty}$.

5. Type $R$ solvmanifolds and their generalizations. Let us begin by defining type $R$ solvable groups. Let $R$ be a connected, simply connected, solvable Lie group with semisimple splitting $R_{s}=M_{R} \rtimes T_{R}$. Then $R$ is said to be of type $R$ if all the eigenvalues of $T_{R}$ have absolute value 1 . (Note. A solvable Lie group is of type $R$ and type $E$ if and only if it is nilpotent.) Let us begin this section with a proof of a theorem on the structure of presentations of compact solvmanifolds $R / H$ where $R$ is a type $R$ solvable Lie group. (See Theorem 4.2 of $[\mathrm{A}, \mathrm{L} ; 4]$.)

Since $T_{R}$ is a subgroup of the orthogonal group if $R$ is type $R$ its closure $T_{R}^{*}$ is compact. Let $R_{s}^{*}=M_{R} \rtimes T_{R}^{*}$. If $R / H$ is the presentation of a compact solvmanifold then $T_{R}=T_{R}^{*}$ and $H / H \cap M_{R}$ is finite.

We may see this as follows. Since $R / H$ is compact and $R_{s}^{*}=R \rtimes C$, where $C$ is a compact group, $R_{s}^{*} / H$ is compact. Let $R^{*}$ be the universal covering group of $R_{s}^{*}$ and let $H^{*}$ be the pre-image of $H$ in $R^{*}$. Then $R^{*}=V \ltimes M_{R}$, where $V$ is the vector space covering group of $T_{R}^{*}$. Now the nil-radical of $R^{*}$ is easily seen to be $M_{R}$. Hence, by the Mostow structure theorem, $M_{R} / H^{*} \cap M_{R}$ is compact and the identity component of $H^{*}, H_{0}^{*}$, is contained in $H^{*} \cap M_{R}$. Further, the image of $H^{*}$ in $R^{*} / M_{R}=V$ is a lattice and so discrete. Hence $H / H \cap M_{R}$ must be finite. Hence $H^{*} \cap M_{R}$ is cocompact in $M_{R}$ and the theorem is now trivial to verify.

It now follows easily that if $R / H$ is the presentation of a compact solvmanifold with $R$ of type $R$ then $R / H$ is finite covered by a nil-manifold of the form $M_{R} / H^{\#}$ where $M_{R}$ is the nil-shadow of $R$ and $H^{\#}$ is of finite index in $H$.

These results may be looked at from a different point of view that leads to the relation between our results and some classical results of Bieberbach and Zassenhaus. Let $M$ be a connected, simply connected nilpotent Lie group and $C$ a compact abelian group of automorphisms of $M$. Form $M \rtimes C$ and let $\Gamma$ be a discrete subgroup of $M \rtimes C$. Then our results above assure us that $M / M \cap \Gamma$ is compact and $\Gamma / M \cap \Gamma$ is finite. It is reasonable to expect this result to be true if we drop the hypothesis that $C$ is abelian and only require that $C$ be a compact group of automorphisms of $M$. This was verified in $[A, L ; 2]$ and a simple proof of the classical theorems of Bieberbach (the special case where $M$ is abelian) was given in $[\mathrm{A}, \mathrm{L} ; 9]$.

The main tool theorem in $[\mathrm{A}, \mathrm{L} ; 2]$ is the following: 
Let $R$ be a connected, simply connected solvable Lie group and let $C$ be a compact group of automorphism of $R$. Further, let $\Gamma$ be a discrete subgroup of $R \rtimes C$ and let $\overline{\Gamma R}$ denote the closure of the group generated by the elements of $\Gamma$ and $R$. Then the identity component of $\overline{\Gamma R}$ is solvable.

In $[A, L ; 1]$, we re-examined the above tool theorem to try and remove the hypothesis that $C$ is compact. We then obtained the following result.

Let $G$ be a Lie group with radical $R$. Further let $\Gamma$ be a discrete subgroup of $G$. Then the identity component of $\overline{\Gamma R}$ is solvable.

We will content ourselves with the statements of these results and how they relate to the theory of solvmanifolds. We do not know how to improve on the proofs in the original articles and so will refer the interested reader to these papers.

\section{REFERENCES}

$[\mathrm{A}, \mathrm{L}]$

1. L. Auslander, On radicals of discrete subgroups of Lie groups, Amer. J. Math. 85(1963), 145-150. MR 27 \#2583.

2. , Bieberbach's theorem on space groups and discrete uniform subgroups of Lie groups. II, Amer. J. Math. 83 (1961), 276-280. MR 23 \# A962.

3. , Solvable Lie groups acting on nilmanifolds, Amer. J. Math. 82 (1960), 653-660. MR 23 \# A241.

4. L. Auslander and L. W. Green, G-induced flows, Amer. J. Math. 88 (1966), 43-60. MR 33 \# 7456.

5. L. Auslander and J. Brezin, Almost algebraic Lie algebras, J. Algebra 8(1968), 295-313. MR 37 \# 344.

6. L. Auslander, Fundamental groups of compact solvmanifolds, Amer. J. Math. 82 (1960), 689-697. MR 23 \# A242.

7. - On a problem of Philip Hall, Ann. of Math. (2) 86 (1967), 112-116. MR 36 $\# 1540$.

8. L. Auslander and R. Tolimieri, Splitting theorems and the structure of solvmanifolds, Ann. of Math. (2) 92 (1970), 164-173. MR 43 \#2733.

9. L. Auslander, An account of the theory of crystallographic groups, Proc. Amer. Math. Soc. 16 (1965), 1230-1236. MR 32 \# 2482.

10. - Discrete uniform subgroups of solvable Lie groups, Trans. Amer. Math. Soc. 99 (1961), 398-402. MR 24 \# A1340.

11. - A characterization of discrete solvable matrix groups, Bull. Amer. Math. Soc. 67 (1961), 235-236. MR 23 \# A945.

12. L. Auslander and M. Auslander, Solvable Lie groups and locally euclidean Riemann spaces, Proc. Amer. Math. Soc. 9 (1958), 933-941. MR 21 \#2021.

13. L. Auslander and R. Tolimieri, On a conjecture of G. D. Mostow and the structure of solvmanifolds, Bull. Amer. Math. Soc. 75 (1969), 1330-1333. MR 40 \# 8077.

14. L. Auslander, The automorphism group of a polycyclic group, Ann. of Math. (2) 89 (1969), 314-322. MR 42 \#6085.

15. - On a problem of Philip Hall (unpublished notes).

16. - Some compact solvmanifolds and locally affine spaces, J. Math. Mech. 7 (1958), 963-975. MR 21 \#93.

17. L. Auslander, et al., Flows on homogeneous spaces, Ann. of Math. Studies, no. 53, Princeton Univ. Press, Princeton, N.J., 1963. MR 29 \#4841.

$[\mathrm{B}, \mathrm{G}]$

G. Birkhoff, Representability of Lie algebras and Lie groups by matrices, Ann. of Math. 38 (1937), 526-532.

$[\mathrm{B}, \mathrm{A}]$ $[\mathrm{D}, \mathrm{J}]$

A. Borel, Linear algebraic groups, Benjamin, New York, 1969. MR 40 \# 4273.

J. Dixmier, L'application exponentielle dans les groupes de Lie résolubles, Bull. Soc. Math. France 85 (1957), 113-121. MR 19, 1182. 
$[\mathrm{J}, \mathrm{N}]$

N. Jacobson, Lie algebras, Interscience Tracts in Pure and Appl. Math., no. 10, Interscience, New York, 1962. MR 26 \# 1345.

$[\mathbf{J}, \mathbf{R}]$

R. W. Johnson, Presentation of solvmanifolds, Amer. J. Math. 94 (1972), 82-102. $[\mathrm{M}, \mathrm{A}]$

1. A. I. Mal'cev, On a class of homogeneous spaces, Izv. Akad. Nauk SSSR Ser. Mat. 13 (1949), 9-22; English transl., Amer. Math. Soc. Transl. (1) 9 (1962), 276-307. MR 10, 507.

2. - Solvable Lie algebras, Izv. Akad. Nauk SSSR Ser. Mat. 9 (1945), 329-352; English transl., Amer. Math. Soc. Transl. (1) 9 (1962), 228-262. MR 9, 173.

3. - On certain classes of infinite solvable groups, Mat. Sb. 28 (70) (1951), 567-588; English transl., Amer. Math. Soc. Transl. (2) 2 (1956), 1-21. MR 12, 203; MR 17, 824.

$[\mathrm{M}, \mathrm{C}]$

C. C. Moore, Decomposition of unitary representations defined by discrete subgroups of nilpotent groups, Ann. of Math. (2) 82 (1965), 146-182. MR 31 \# 5928.

$[\mathrm{M}, \mathrm{G}]$

1. G. D. Mostow, Factor spaces of solvable groups, Ann. of Math. (2) 60 (1954), 1-27. MR 15, 853 .

2. , Fully reducible subgroups of algebraic groups, Amer. J. Math. 78 (1956), 200-201. MR 19, 1181.

3. - Representative functions on discrete groups and solvable arithmetic subgroups, Amer. J. Math. 92 (1970), 1-32. MR 42 \#6150.

4. - Some applications of representative functions to solvmanifolds, Amer. J. Math. 93 (1971), 11-32.

5. - , Arithmetic subgroups of groups with radical, Ann. of Math. (2) 93 (1971), $409-438$.

6. - On the fundamental group of a homogeneous space, Ann. of Math. (2) 66 (1957), 249-255; Errata, 590. MR 19, 561; 752.

7. MR 26 \#256.

8. - Homogeneous spaces with finite invariant measure, Ann. of Math. (2) 75 (1962), 17-37. MR 26 \#2546.

9. - The rigidity of locally symmetric spaces, Actes Congrès Intern. Math., 1970, tome 2, pp. 187-197.

$[\mathrm{S}, \mathrm{M}]$

M. Saito, Sur certains groupes de Lie résolubles, Sci. Papers Coll. Gen. Ed. Univ. Tokyo 7 (1957), 1-11, 157-168. MR 20 \#3931; \#3932.

$[\mathrm{S}, \mathrm{J}]$

J. Scheuneman, Two-step nilpotent Lie algebras, J. Algebra 7 (1967), 152-159. MR 36 $\# 225$.

$[\mathrm{T}, \mathrm{R}]$

1. R. Tolimieri, Structure of solvable Lie algebras, J. Algebra 16 (1970), 597-625.

2. - Applications of the semi-simple splitting, Bull. Amer. Math. Soc. 77 (1971), 275-280. MR 42 \# 7823.

3. ㄴ. On the Selberg condition for subgroups of a solvable Lie group, Bull. Amer. Math. Soc. 77 (1971), 584-586.

4. — , Finite invariant measure, Geometriae Dedicata 1 (1972).

5. - Notes on nilpotent groups (unpublished).

$[\mathrm{W}, \mathrm{H}]$

H. C. Wang, Discrete subgroups of solvable Lie groups. I, Ann. of Math. (2) 64 (1956), 1-19. MR 17, 1224.

Department of Mathematics, Institute for Advanced Study, Princeton, New JERSEY 08540

Graduate Center, City University of New York, New York, New York 10036 (Current address) 